\title{
PSEUDOLOCALITY FOR THE RICCI FLOW AND APPLICATIONS
}

\section{INTRODUCTION}

In this article we consider the Ricci flow

$$
\frac{\partial}{\partial t} g_{i j}=-2 R_{i j}
$$

on a complete non-compact Riemannian manifold $(M, g)$, and the heat equation and conjugate heat equation

$$
\begin{gathered}
\frac{\partial u}{\partial t}-\Delta^{t} u=0 \\
\frac{\partial u}{\partial t}+\Delta^{t} u-R u=0
\end{gathered}
$$

where $\Delta^{t}$ denotes the Laplacian operator with respect to a solution $g(t)$ to (1.1), and $R(t)$ is the scalar curvature of $g(t)$. Notice that if $g(t)$ is

Date: December, 2006.

2000 Mathematics Subject Classification. Primary 53C44; Secondary 58J37, 35B35.

${ }^{1}$ Research partially supported by NSERC grant no. \# 327637-06.

${ }^{2}$ Research partially supported by Earmarked Grant of Hong Kong \#CUHK403005. 
defined on an interval $[0, T]$ and we let $\tau=T-t$, then (1.3) defines a strictly parabolic equation on $M$ with respect to $\tau \in[0, T]$.

The conjugate heat equation corresponding to the Ricci flow was considered in [26], and there Perelman established a differential Li-YauHamilton (LYH) type inequality for its fundamental solutions ([26]; Corollary 9.3) on compact manifolds. The proof was sketched in [26] and a detailed proof was given by $\mathrm{Ni}$ in [23]. As an application of the LYH inequality, Perelman proved a pseudolocality result for the Ricci flow on compact manifolds ([26]; Theorem 10.1), which basically states that regions of large amounts of curvature cannot instantly affect almost Euclidean regions under the Ricci flow. For more details of the proof, see [7, 16, 28].

In this article we verify these results, including the LHY hamilton inequality and pseudolocality, in the case of complete non-compact Riemannian manifolds. We basically follow the original steps described in [26] as well as those in [7, 16, 28, 23].

Our motivation to generalize Perelman's results mentioned above is to study long time existence of Ricci flow and Kähler-Ricci flow on complete noncompact manifold. Using the result on pseudolocality, we obtain the following:

Theorem 1.1. Let $\left(M^{n}, g\right)$ be a complete noncompact Riemannian manifold with injectivity radius bounded away from zero such that

$$
|R m|(x) \rightarrow 0
$$

as $x \rightarrow \infty$. Let $(M, g(t))$ be the corresponding maximal solution to the Ricci flow (9.1) on $M \times[0, T)$. Then either $T=\infty$ or there exists some compact $S \subset M$ with the property that $|R m(x, t)|$ is bounded on $(M \backslash S) \times[0, T)$.

The conditions are satisfied if $M$ is an asymptotically flat manifold for example. As a Corollary to Theorem 1.1 we also have

Corollary 1.1. Suppose $T<\infty$ in Theorem 1.1. Then $\operatorname{Rm}(x, T) \rightarrow 0$ as $x \rightarrow \infty$ in the sense that: given any $\epsilon>0$, we may choose $S$ such that $|\operatorname{Rm}(x, t)| \leq \epsilon$ for all $(x, t) \in S^{c} \times[0, T)$.

Combining Theorem 1.1 with the results in [25], we have the following result on the long time existence of Kähler-Ricci on complete noncompact Kähler manifolds with nonnegative holomorphic bisectional curvature. 
Theorem 1.2. Let $\left(M^{n}, g_{0}\right)$ be a complete non-compact Kähler manifold with non-negative holomorphic bisectional curvature with injectivity radius bounded away from zero such that

$$
|R m|(x) \rightarrow 0
$$

as $x \rightarrow \infty$. Then the Kähler-Ricci flow

$$
\frac{\partial}{\partial t} g_{i \bar{j}}=-R_{i \bar{j}}
$$

with initial data $g_{0}$ has a long time solution $g(t)$ on $M \times[0, \infty)$.

The Kähler-Ricci flow is an important tool to study uniformization of complete noncompact Kähler manifolds with nonnegative holomorphic bisectional curvature, see [34, 1, 2] for example. In [34] (see also [25]), it was proved that if $\left(M^{n}, g_{0}\right)$ is a complete non-compact Kählermanifold with non-negative and bounded holomorphic bisectional curvature, and if the scalar curvature satisfies:

$$
\frac{1}{V_{x}(r)} \int_{B_{x}(r)} R \leq \frac{C}{1+r^{\theta}}
$$

for some $C, \theta>0$ for all $x$ and $r$, then (1.4) has long time solution. By the result in [24], (1.5) is true for $\theta=1$, at least for simply connected $M$ and where the constant $C$ which may depend on $x$. It is unclear whether (1.5) is true in general with $C$ being independent of $x$ except for the case of maximal volume growth, see [22].

In order to prove the LYH type differential inequality for the fundamental solution of (1.2), we need to obtain estimates for the fundamental solution together with some gradient estimates for positive solutions of (1.2) and (1.3). In case the manifold is compact, results have been obtained by Zhang, Kuang-Zhang [35, 17] and Ni [23]. Some estimates are also obtained for complete manifolds with nonnegative Ricci curvature by $\mathrm{Ni}$ [21]. We consider the case that the manifold is complete, non-compact, and has bounded curvature. The results may have independent interest.

The paper is organized as follows. In every section, our results are obtained on a complete non-compact Riemannian manifold. In $\S 2-\S 4$ we establish some basic estimates for positive solutions of the conjugate heat equation associated to a general evolution (2.1) of a Riemannian metric. In $\S 5$ and $\S 6$ we establish estimates for fundamental solutions of this conjugate heat equation. In $\S 7$ we apply our previous estimates to establish the LYH inequality for the fundamental solution of the conjugate heat equation associated to the Ricci flow (1.1). Our steps in this 
section basically follow the steps in [23] 1]. In $\S 8$ we establish pseudolocality for the Ricci flow (1.1) on complete non-compact Riemannian manifolds. In particular, we show that Theorem 10.1 in [26] holds in the non-compact case. In $\S 9$ we prove Theorem 1.1 and Theorem 1.2 .

\section{AN INTEGRAL ESTIMATE}

In this section, we will modify the arguments by Grigor'yan [11 a little bit to obtain an integral estimate for solution of (2.2). The proof is basically the same as in [11].

Let $\{g(t) \mid t \in[0, T]\}$ be a smooth family of complete Riemannian metrics on $M^{n}$ such that $g(t)$ satisfies:

$$
\frac{\partial}{\partial t} g_{i j}(x, t)=2 h_{i j}(x, t)
$$

on $M \times[0, T]$, where $h_{i j}(x, t)$ is a smooth family of symmetric tensors.

Consider the equation:

$$
\frac{\partial u}{\partial t}-\Delta^{t} u+q u=0
$$

where $\Delta^{t}$ denotes the Laplacian operator with respect to $g(t)$ and $q$ is a smooth function on $M \times[0, T]$

Let us make the following assumptions:

(A1) $\|h\|,\left\|\nabla^{t} h\right\|$ are uniformly bound on space-time, where the norm is taken with respect to $t$.

(A2) The sectional curvatures of the metrics $g(t)$ are uniformly bounded on space-time.

(A3) $|q|,|| \nabla^{t} q||,\left|\Delta^{t} q\right|$ are uniformly bounded on space-time.

Let $H(t)$ be the trace of $h_{i j}(t)$ with respect to $g(t)$.

Definition 2.1. Let $f$ be a positive function on $(0, T]$. $f$ is said to be regular with the constants $\gamma>1$ and $A \geq 1$, if

(i) $f$ is increasing, and

(ii) $\frac{f(s)}{f(s / \gamma)} \leq A \frac{f(t)}{f(t / \gamma)}$ for any $0<s \leq t \leq T$.

Lemma 2.1. Let $\Omega$ be a relative compact domain of $M$ with smooth boundary and let $K$ be a compact set with $K \subset \subset \Omega$. Let $u$ be any

\footnotetext{
${ }^{1}$ Our proof does not use the reduced distance $L(y, \tau)$ associated to the Ricci flow, introduced in [26])
} 
solution to the problem:

$$
\left\{\begin{array}{l}
u_{t}-\Delta^{t} u+q u=0, \text { in } \Omega \times[0, T] \\
\left.u\right|_{\partial \Omega \times[0, T]}=0 \\
\operatorname{supp} u(\cdot, 0) \subset K .
\end{array}\right.
$$

Let $f$ be a regular function with the constants $\gamma$ and $A$. Suppose

$$
\int_{\Omega} u^{2} d V_{t} \leq \frac{1}{f(t)}
$$

for any $t>0$. Then there is a positive constant $C$ depending only on $\gamma$, the uniform upper bound of $|q|$ and $|H|$, and a positive constant $D$ depending only on $T, \gamma$ and the uniform upper bound of $\|h\|$, such that

$$
\int_{\Omega} u^{2}(x, t) e^{\frac{r^{2}(x, K)}{D t}} d V_{t} \leq \frac{4 A}{f(t / \gamma)} e^{C t}
$$

for any $t>0$, where $r(x, K)$ denotes the distance between $x$ and $K$ with respect to the initial metric.

Proof. The proof is almost the same as the proof of Theorem 2.1 in Grigoryan [11].

Let $C_{2}>0$ be a constant such that $|q|+1 / 2|H| \leq C_{2}$, and let $v=e^{-C_{2} t} u$. Then $v$ satisfies

$$
v_{t}-\Delta^{t} v+\left(C_{2}+q\right) v=0
$$

and

$$
\int_{\Omega} v^{2} d V_{t}=e^{-2 C_{2} t} \int_{\Omega} u^{2} d V_{t} \leq \frac{1}{f(t) e^{2 C_{2} t}}:=\frac{1}{\tilde{f}(t)}
$$

where $\tilde{f}(t)=f(t) e^{2 C_{2} t}$ is regular with constants $\gamma$ and $A$.

Now for any $R>0$, define

$$
d(x)= \begin{cases}R-r(x, K) & x \in K^{R} \\ 0 & x \notin K^{R}\end{cases}
$$

where $K^{R}$ means the $R$-neighborhood of $K$ with respect to the initial metric. Then $\left|\nabla^{t} d\right| \leq C_{1}$ uniformly on space-time where $C_{1}$ depends on $T$ and the upper bound of $\|h\|$. Then if we let $\xi(x, s-t)=\frac{d^{2}(x)}{2 C_{1}^{2}(t-s)}$ for $s>T$ fixed and $0<t \leq T<s$, we have

$$
\frac{\partial}{\partial t} \xi+\frac{1}{2}\left|\nabla^{t} \xi\right|^{2}=-\frac{d^{2}}{2 C_{1}^{2}(t-s)^{2}}+\frac{1}{2} \frac{d^{2}\left\|\nabla^{t} d\right\|^{2}}{C_{1}^{4}(t-s)^{2}} \leq 0,
$$


which combines with (2.4) to give

$$
\begin{aligned}
& \frac{d}{d t} \int_{\Omega} v^{2} e^{\xi} d V_{t} \\
= & \int_{\Omega}\left(2 v v_{t} e^{\xi}+v^{2} e^{\xi} \xi_{t}\right) d V_{t}+\int_{\Omega} v^{2} e^{\xi} H d V_{t} \\
\leq & 2 \int_{\Omega} v\left(\Delta^{t} v-\left(C_{2}+q\right) v\right) e^{\xi} d V_{t}-\frac{1}{2} \int_{\Omega} v^{2} e^{\xi}\left|\nabla^{t} \xi\right|^{2} d V_{t}-\int_{\Omega} H v^{2} e^{\xi} d V_{t} \\
\leq & -2 \int_{\Omega}\left|\nabla^{t} v\right|^{2} e^{\xi} d V_{t}-2 \int_{\Omega} v e^{\xi}\left\langle\nabla^{t} v, \nabla^{t} \xi\right\rangle d V_{t}-\frac{1}{2} \int_{\Omega} v^{2} e^{\xi}\left|\nabla^{t} \xi\right|^{2} d V_{t} \\
\leq & 0,
\end{aligned}
$$

where we have used the fact that $v=0$ on $\partial \Omega, 2 C_{2}+2 q+H \geq 0$ and (2.5).

It now follows from STEP1 and STEP2 of the proof of Theorem 2.1 in Griyor'yan [11], that there exists a positive constant $D>0$ depending on $C_{1}$ and $\gamma$, such that

$$
\int_{\Omega} v^{2} e^{\frac{r^{2}(x, K)}{D t}} d V_{t} \leq \frac{4 A}{\tilde{f}(t / \gamma)} .
$$

Thus,

$$
\int_{\Omega} u^{2} e^{\frac{r^{2}(x, K)}{D t}} d V_{t} \leq \frac{4 A}{f(t / \gamma)} e^{2 C_{2}\left(1-\frac{1}{\gamma}\right) t}
$$

\section{A mean VAlue inequality}

In this section, we prove the following lemma which will be used to estimate the fundamental solution of (6.3).

Lemma 3.1. Let $u$ be a positive sub-solution of equation (2.2) on $\Omega \times$ $[0, T]$ where $\Omega$ is a domain in $M$. Moreover, suppose that there is a complete Riemannian metric $\tilde{g}$ on $M$ with Ricci curvature bounded from below by $-k$ with $k \geq 0$, such that

$$
\frac{1}{C_{0}} \tilde{g} \leq g(0) \leq C_{0} \tilde{g}
$$

in $\Omega$ for some $C_{0}>0$. Let $\tilde{Q}_{r}(x, t):=\tilde{B}_{x}(r) \times\left(t-r^{2}, t\right]$ whenever it is well defined, where $\tilde{B}_{x}(r)$ means the ball of radius $r$ with respect to $\tilde{g}$. Then for any $(x, t) \in \Omega \times(0, T]$ and $r>0$ such that $\tilde{Q}_{2 r}(x, t) \subset \subset$ $\Omega \times[0, T]$

$$
\sup _{\tilde{Q}_{r}(x, t)} u \leq \frac{C e^{A t+B \sqrt{k} r}}{r^{2} \tilde{V}_{x}(r)} \int_{\tilde{Q}_{2 r}(x, t)} u d \tilde{V} d s
$$


where $A$ depends only on the the upper bounds of $|q|$ and $|H|$ on $\Omega$, $B$ depends only on $n$, and $C$ depends only on $C_{0}, n, T$ and the uniform upper bound of $|h|$ on $\Omega$. The notations $\tilde{V}_{x}(r)$ and $d \tilde{V}$ denote the volumes with respect to $\tilde{g}$.

Proof. The proof is almost the same as in Zhang [[35]; § 5]. We just emphasis on our modifications. For details, please refer to the paper.

Let $\sigma$ be in $(1,2]$. Let $\phi$ be a smooth function on $[0, \infty)$, such that: i) $\phi=1$ on $[0, r]$, ii) $\phi=0$ on $[\sigma r, \infty)$ and iii) $-\frac{2}{(\sigma-1) r} \leq \phi^{\prime} \leq 0$.

Let $\eta$ be a smooth function on $[0, \infty)$, such that: i) $\eta=0$ on $[0, t-$ $\left.\sigma^{2} r^{2}\right]$, ii) $\eta=1$ on $\left[t-r^{2}, \infty\right)$ and iii) $0 \leq \eta^{\prime} \leq \frac{2}{(\sigma-1)^{2} r^{2}}$.

Let $\psi(y, s)=\phi(\tilde{r}(x, y)) \eta(s)$ where $x$ is fixed and $\tilde{r}(x, y)$ means the distance function of $\tilde{g}$. Then $\operatorname{supp} \psi(\cdot, s) \subset \overline{\tilde{B}_{x}(2 r)} \subset \Omega$.

Let $v=e^{-C_{1} t} u$, where $C_{1}$ is some positive constant to be determined. Then

$$
v_{t}-\Delta^{t} v+\left(C_{1}+q\right) v \leq 0 .
$$

For any $p \geq 1$,

$$
\frac{\partial v^{p}}{\partial t}-\Delta^{t}\left(v^{p}\right)+p\left(C_{1}+q\right) v^{p} \leq 0 .
$$

Let $w=v^{p}$. Let $t^{\prime}$ be any real number in $\left[t-r^{2}, t\right]$. Multiply $w \psi^{2}$ to the inequality above and integrate. We get

$$
\begin{gathered}
-\int_{t-\sigma^{2} r^{2}}^{t^{\prime}} \int_{M} \psi^{2} w \Delta^{s} w d V_{s} d s+p \int_{t-\sigma^{2} r^{2}}^{t^{\prime}} \int_{M}\left(C_{1}+q\right) \psi^{2} w^{2} d V_{s} d s \\
\leq-\int_{t-\sigma^{2} r^{2}}^{t^{\prime}} \int_{M} \psi^{2} w w_{s} d V_{s} d s
\end{gathered}
$$

Integrating by parts in the first term on the left in the above inequality, we get

$$
\begin{aligned}
-\int_{t-\sigma^{2} r^{2}}^{t^{\prime}} \int_{M} \psi^{2} w \Delta^{s} w d V_{s} d s & =\int_{t-\sigma^{2} r^{2}}^{t^{\prime}} \int_{M}\left\langle\nabla^{s}\left(\psi^{2} w\right), \nabla^{s} w\right\rangle_{s} d V_{s} d s \\
& =\int_{t-\sigma^{2} r^{2}}^{t^{\prime}} \int_{M}\left(\left|\nabla^{s}(\psi w)\right|^{2}-\left|\nabla^{s} \psi\right|^{2} w^{2}\right) d V_{s} d s .
\end{aligned}
$$


Moreover, the second term on the left in (3.2) is:

$$
\begin{aligned}
-\int_{t-\sigma^{2} r^{2}}^{t^{\prime}} & \int_{M} \psi^{2} w w_{s} d V_{s} d s \\
= & -\int_{t-\sigma^{2} r^{2}}^{t^{\prime}} \int_{M} \psi^{2} w w_{s} e^{F} d V d s \\
= & -\int_{M} \int_{t-\sigma^{2} r^{2}}^{t^{\prime}} \frac{1}{2}\left[\left(\psi^{2} w^{2} e^{F}\right)_{s}-\left(\psi^{2} e^{F}\right)_{s} w^{2}\right] d s d V \\
= & -\frac{1}{2} \int_{M} w^{2} \psi^{2} d V_{t^{\prime}}+\int_{t-\sigma^{2} r^{2}}^{t^{\prime}} \int_{M} \psi \psi_{s} w^{2} d V_{s} d s \\
& +\frac{1}{2} \int_{t-\sigma^{2} r^{2}}^{t^{\prime}} \int_{M} H \psi^{2} w^{2} d V_{s} d s
\end{aligned}
$$

where $F$ is such that $e^{F} d V=d V_{s}$ and $d V$ is the volume element for $g(0)$.

Choose $C_{1}$ large enough depending only on the uniform upper bounds of $|q|$ and $|H|$ on $\Omega$. Then by (3.2)-(3.4), we have:

$$
\begin{array}{r}
\int_{t-\sigma^{2} r^{2}}^{t^{\prime}} \int_{M}\left|\nabla^{s}(\psi w)\right|^{2} d V_{s} d s+\frac{1}{2} \int_{M} w^{2} \psi^{2} d V_{t^{\prime}} \\
\leq \int_{t-\sigma^{2} r^{2}}^{t^{\prime}} \int_{M}\left(\psi \psi_{s}+\left|\nabla^{s} \psi\right|^{2}\right) w^{2} d V_{s} d s
\end{array}
$$

Note that

$$
\psi_{s}=\phi \eta^{\prime} \leq \frac{2}{(\sigma-1)^{2} r^{2}}
$$

Moreover

$$
\left|\nabla^{s} \psi\right|^{2}=\eta^{2}\left(\phi^{\prime}\right)^{2}\left|\nabla^{s}(\tilde{r}(x, \cdot))\right|^{2} \leq \frac{C_{2}}{(\sigma-1)^{2} r^{2}}
$$

where $C_{2}$ depends on $C_{0}, T$ and the uniformly upper bound of $|h|$ on $\Omega$.

Hence

$$
\begin{aligned}
& \int_{t-\sigma^{2} r^{2}}^{t^{\prime}} \int_{\tilde{B}_{x}(\sigma r)}\left|\nabla^{s}(\psi w)\right|^{2} d V_{s} d s+\frac{1}{2} \int_{M} w^{2} \psi^{2} d V_{t^{\prime}} \\
\leq & \frac{C_{3}}{(\sigma-1)^{2} r^{2}} \int_{t-\sigma^{2} r^{2}}^{t^{\prime}} \int_{\tilde{B}_{x}(\sigma r)} w^{2} d V_{s} d s
\end{aligned}
$$

where $C_{3}=\max \left\{2, C_{2}\right\}$. So,

$$
\int_{t-\sigma^{2} r^{2}}^{t} \int_{\tilde{B}_{x}(\sigma r)}|\tilde{\nabla}(\psi w)|^{2} d \tilde{V} d s \leq \frac{C_{4}}{(\sigma-1)^{2} r^{2}} \int_{t-\sigma^{2} r^{2}}^{t} \int_{\tilde{B}_{x}(\sigma r)} w^{2} d \tilde{V} d s \text { and }
$$




$$
\max _{t-r^{2} \leq t^{\prime} \leq t} \int_{\tilde{B}_{x}(\sigma r)} w^{2} \psi^{2} d \tilde{V} \leq \frac{C_{4}}{(\sigma-1)^{2} r^{2}} \int_{t-\sigma^{2} r^{2}}^{t} \int_{\tilde{B}_{x}(\sigma r)} w^{2} d \tilde{V} d s
$$

where $C_{4}$ depends only on $C_{0}, T$ and the uniform upper bound of $|h|$.

Now we can proceed as in the proof of Theorem 5.1 in 35] with respect to the metric $\tilde{g}$. Applying the the Sobolev inequality in [27] with respect to $\tilde{g}$ and the Moser iteration as in Zhang [35], we get

$$
\sup _{\tilde{Q}_{r}(x, t)} v^{2} \leq \frac{C_{5} e^{C_{6} \sqrt{k} r}}{r^{2} \tilde{V}_{x}(r)}\left(\frac{1}{\log \gamma}\right)^{\frac{n+2}{2}} \int_{\tilde{Q}_{\gamma r}(x, t)} v^{2} d \tilde{V} d s
$$

for any $\gamma \in(1,2]$, where $C_{5}$ depends only on $C_{0}, T$ the uniform upper bound of $|h|$ and $n$, and $C_{6}$ depends only $n$.

By the trick on iteration of Li-Schoen [18] as mentioned in [35], we get

$$
\sup _{\tilde{Q}_{r}(x, t)} v \leq \frac{C_{7} e^{C_{8} \sqrt{k} r}}{r^{2} \tilde{V}_{x}(r)} \times \frac{1}{\log ^{\frac{n+2}{2}} \gamma} \int_{\tilde{Q}_{\gamma r}(x, t)} v d \tilde{V} d s
$$

for any $\gamma \in(1,2]$, where $C_{7}$ depends only on $C_{0}, T$ the upper bound of $|h|$ and $n$, and $C_{8}$ depends only on $n$.

In particular, let $\gamma=2$, we get

$$
\sup _{\tilde{Q}_{r}(x, t)} v \leq \frac{C_{9} e^{C_{8} \sqrt{k} r}}{r^{2} \tilde{V}_{x}(r)} \int_{\tilde{Q}_{2 r}(x, t)} v d \tilde{V} d s
$$

where $C_{9}$ depends only on $C_{0}, T$ the uniform upper bound of $|h|$ and $n$. So

$$
\begin{aligned}
\sup _{\tilde{Q}_{r}(x, t)} u & \leq e^{C_{1} t} \sup _{\tilde{Q}_{r}(x, t)} v \\
& \leq \frac{C_{9} e^{C_{1} t+C_{8} \sqrt{k} r}}{r^{2} \tilde{V}_{x}(r)} \int_{\tilde{Q}_{2 r}(x, t)} u d \tilde{V} d s .
\end{aligned}
$$

\section{A Li-Yau type GRadient estimate}

In this section, we derive a Li-Yau type graident estimate which will also be used in the estimates of the fundamental solution of 7.3 . We basically follow the proof in [19]. Let $g(t)$ be as in $\S 2$.

Lemma 4.1. Let $u$ be a positive solution to equation (2.2). Then for any $\alpha>1$ and $\epsilon>0$, there is a constant $C>0$ depending on $\alpha, \epsilon, n, T$, 
the uniform upper bounds of $|h|,|\nabla h|,|\nabla q|,|\Delta q|,\left|R c^{t}\right|$ and the bound of the sectional curvature at $t=0$, such that

$$
\frac{|\nabla u|^{2}}{u^{2}}-\alpha \frac{u_{t}}{u}-\alpha q \leq C+\frac{(n+\epsilon) \alpha^{2}}{2 t}
$$

Proof. In the following $\nabla$ and $\Delta$ are understood to be time dependent. For any smooth function $f$ on $M \times[0, T)$, at a point with normal coordinates with respect to the metric $g(t)$, we have

$$
\begin{aligned}
(\Delta f)_{t} & =\Delta f_{t}-2 h_{i j} f_{i j}-2 h_{i k ; i} f_{k}+H_{i} f_{i} \\
\left(|\nabla f|^{2}\right)_{t} & =2\left\langle\nabla f_{t}, \nabla f\right\rangle-2 h(\nabla f, \nabla f) .
\end{aligned}
$$

Repeated indices mean summation.

Let $f=\log u$. Then

$$
\Delta f-f_{t}=q-|\nabla f|^{2}
$$


For $\alpha>1$ and $\epsilon>0$, let $F=t\left(|\nabla f|^{2}-\alpha f_{t}-\alpha R\right)$. Then in normal coordinates

$$
\begin{aligned}
\Delta F & =t\left[2 \sum_{i j} f_{i j}^{2}+2\langle\nabla(\Delta f), \nabla f\rangle+2 R_{i j} f_{i} f_{j}-\alpha(\Delta f)_{t}\right. \\
& \left.-2 \alpha h_{i j} f_{i j}-2 \alpha h_{i k ; i} f_{k}+\alpha H_{i} f_{i}-\alpha \Delta q\right] \\
& =t\left(2 \sum_{i j} f_{i j}^{2}-2 \alpha h_{i j} f_{i j}\right)+2 t\left\langle\nabla\left(f_{t}+q-|\nabla f|^{2}\right), \nabla f\right\rangle \\
& -\alpha t\left(f_{t}+q-|\nabla f|^{2}\right)_{t}-\alpha t\left(2 h_{k i ; k}-H_{i}\right) f_{i}+2 t R_{i j} f_{i} f_{j}-\alpha t \Delta q \\
& =t\left(2 \sum_{i j} f_{i j}^{2}-2 \alpha h_{i j} f_{i j}\right)+2 t\left\langle\nabla\left(f_{t}+q-|\nabla f|^{2}\right), \nabla f\right\rangle \\
& +\alpha t\left(\frac{1}{\alpha} \frac{F}{t}+\left(1-\frac{1}{\alpha}\right)|\nabla f|^{2}\right)_{t}-\alpha t\left(2 h_{k i ; k}-H_{i}\right) f_{i}+2 t R_{i j} f_{i} f_{j}-\alpha t \Delta q \\
& =t\left(2 \sum_{i j} f_{i j}^{2}-2 \alpha h_{i j} f_{i j}\right)+2 t\left\langle\nabla\left(\alpha f_{t}+q-|\nabla f|^{2}\right), \nabla f\right\rangle \\
& +F_{t}-\frac{F}{t}-2 t(\alpha-1) h_{i j} f_{i} f_{j}-\alpha t\left(2 h_{k i ; k}-H_{i}\right) f_{i}+2 t R_{i j} f_{i} f_{j}-\alpha t \Delta q \\
& =t\left(2 \sum_{i j} f_{i j}^{2}-2 \alpha h_{i j} f_{i j}\right)-2 t\left\langle\nabla\left(\frac{F}{t}+(\alpha-1) q\right), \nabla f\right\rangle \\
& +F_{t}-\frac{F}{t}-2 t(\alpha-1) h_{i j} f_{i} f_{j}-\alpha t\left(2 h_{k i ; k}-H_{i}\right) f_{i}+2 t R_{i j} f_{i} f_{j}-\alpha t \Delta q \\
& =t\left(2 \sum_{i j} f_{i j}^{2}-2 \alpha h_{i j} f_{i j}\right)-2\langle\nabla F, \nabla f\rangle+F_{t}-\frac{F}{t} \\
& -2 t(\alpha-1) h_{i j} f_{i} f_{j}-\alpha t\left(2 h_{k i ; k}-H_{i}\right) f_{i}+2 t R_{i j} f_{i} f_{j}-\alpha t \Delta q \\
& -2 t(\alpha-1) q_{i} f_{i} .
\end{aligned}
$$

By [34], there is a smooth function $\rho$ such that

$$
\left\{\begin{aligned}
\frac{1}{C_{1}} \rho(x) & \leq r_{0}(x) \leq C_{1} \rho(x) \\
\left|\nabla^{0} \rho\right| & \leq C_{1} \\
\left|\nabla^{0} \nabla^{0} \rho\right| & \leq C_{1}
\end{aligned}\right.
$$

where $C_{1}$ is a constant depending on $n$ and the bound of $|R m|$ of $g(0)$. Here $r_{0}(x)$ is the distance with respect to $g(0)$ from a fixed point $o$. By the assumption that $|h|$ and $|\nabla h|$ are uniformly bounded on space-time, we have 


$$
\left\{\begin{aligned}
\frac{1}{C_{2}} \rho(x) & \leq r_{t}(x) \leq C_{2} \rho(x) \\
|\nabla \rho| & \leq C_{2} \\
\left|\nabla^{2} \rho\right| & \leq C_{2}
\end{aligned}\right.
$$

where $C_{2}$ depends on $C_{1}, T$ and the uniformly upper bound of $|h|$ and $|\nabla h|$. Here $r_{t}(x)$ is the distance with respect to $g(t)$ from $o$.

Let $\eta \in C^{\infty}([0, \infty)$ be such that $0 \leq \eta \leq 1, \eta=1$ on $[0,1]$ and $\eta=0$ on $[2, \infty), \eta>0$ on $[0,2), 0 \geq \eta^{\prime} / \eta^{\frac{1}{2}} \geq-C_{3}$ and $\eta^{\prime \prime} \geq-C_{3}$ on $[0, \infty)$ where $C_{3}$ is some positive absolute constant. For any $R>$ 0 , let $\phi=\eta(\rho / R)$. Suppose at the point $\left(x_{0}, t_{0}\right)$ where $\phi F$ attains positive maximum, $0<t_{0} \leq T$. Then at $\left(x_{0}, t_{0}\right)$, we have $\phi F_{t} \geq 0$, $F \nabla \phi+\phi \nabla F=0$ and $\Delta(\phi f) \leq 0$. Hence at $\left(x_{0}, t_{0}\right)$ :

$$
\begin{aligned}
0 & \geq \Delta(\phi F) \\
& =\phi \Delta F+2\langle\nabla \phi, \nabla F\rangle+F \Delta \phi \\
& =\phi \Delta F-2 F \frac{|\nabla \phi|^{2}}{\phi}+F \Delta \phi \\
& \geq \phi \Delta F-C_{4} F\left(R^{-1}+R^{-2}\right) \\
& \geq t_{0} \phi\left(2 f_{i j}^{2}-2 \alpha h_{i j} f_{i j}\right)-2 \phi\langle\nabla F, \nabla f\rangle+F_{t}-\phi \frac{F}{t_{0}} \\
& -C_{5} t_{0} \phi|\nabla f|^{2}-C_{5} t_{0} \phi-C_{4} F\left(R^{-1}+R^{-2}\right) \\
& \geq t_{0} \phi\left(2 f_{i j}^{2}-2 \alpha h_{i j} f_{i j}\right)+2 F\langle\nabla \phi, \nabla f\rangle-\phi \frac{F}{t_{0}}+ \\
& -C_{5} t \phi|\nabla f|^{2}-C_{5} t \phi-C_{4} F\left(R^{-1}+R^{-2}\right) \\
& \geq t_{0} \phi\left(2 f_{i j}^{2}-2 \alpha h_{i j} f_{i j}\right)-C_{6} F \phi^{\frac{1}{2}} R^{-1}|\nabla f|-\phi \frac{F}{t_{0}} \\
& -C_{5} t_{0} \phi|\nabla f|^{2}-C_{5} t_{0} \phi-C_{4} F\left(R^{-1}+R^{-2}\right) \\
& \geq t_{0} \phi \cdot \frac{2 n}{n+\epsilon} f_{i j}^{2}-C_{6} F \phi^{\frac{1}{2}} R^{-1}|\nabla f|-\phi \frac{F}{t_{0}} \\
& -C_{5} t_{0} \phi|\nabla f|^{2}-C_{7} t_{0} \phi-C_{4} F\left(R^{-1}+R^{-2}\right) \\
& \geq t_{0} \phi \frac{2}{n+\epsilon}(\Delta f)^{2}-C_{6} F \phi^{\frac{1}{2}} R^{-1}|\nabla f|-\phi \frac{F}{t_{0}} \\
& -C_{5} t_{0} \phi|\nabla f|^{2}-C_{7} t_{0} \phi-C_{4} F\left(R^{-1}+R^{-2}\right) \\
& =t_{0} \phi \frac{2}{n+\epsilon}\left(|\nabla f|^{2}-f_{t}-q\right)^{2}-C_{6} F \phi^{\frac{1}{2}} R^{-1}|\nabla f|-\phi \frac{F}{t_{0}} \\
& -C_{5} t_{0} \phi|\nabla f|^{2}-C_{7} t_{0} \phi-C_{4} F\left(R^{-1}+R^{-2}\right) .
\end{aligned}
$$


Here and below $C_{i}$ 's are constants depending only on bounds of $h,|\nabla h|$, $|R m|$ of $g(0),|\Delta q|, \alpha, \epsilon, n$ and $T$.

Multiply both sides by $t_{0} \phi$, and let $A=\phi|\nabla f|^{2}, B=\phi\left(f_{t}+q\right)$ so that $\phi F=t_{0}(A-\alpha B)$. Then for any $\delta>0$ and $\tau>0$ :

$$
\begin{aligned}
& 0 \geq \frac{2 t_{0}^{2}}{n+\epsilon}(A-B)^{2}-C_{6} t_{0} \phi F R^{-1} A^{\frac{1}{2}}-\phi^{2} F-C_{5} t_{0}^{2} \phi A-C_{7} t_{0}^{2} \phi^{2} \\
& \quad-C_{4} t_{0} \phi F\left(R^{-1}+R^{-2}\right) \\
& \geq \phi F\left[-C_{4} t_{0}\left(R^{-1}+R^{-2}\right)-1\right] \\
& \quad+\frac{2 t_{0}^{2}}{n+\epsilon}\left[(A-B)^{2}-\frac{C_{6}}{2} R^{-1}(A-\alpha B) A^{\frac{1}{2}}-C_{5} A\right]-C_{7} t_{0}^{2} \\
& \geq \phi F\left[-C_{4} t_{0}\left(R^{-1}+R^{-2}\right)-1\right]+\frac{2 t_{0}^{2}}{n+\epsilon}\left[(A-B)^{2}\right. \\
&\left.\quad-\frac{C_{6}}{2 \delta} R^{-2} \delta^{-1}(A-\alpha B)-\delta(A-\alpha B) A-C_{5} A\right]-C_{7} t_{0}^{2} \\
& \geq \phi F {\left[-C_{8} t_{0}\left(1+\delta^{-1}\right)\left(R^{-1}+R^{-2}\right)-1\right] } \\
&+\frac{2 t_{0}^{2}}{n+\epsilon}\left[(A-B)^{2}-\delta(A-\alpha B) A-\tau A^{2}\right]-C_{10}\left(1+\tau^{-1}\right) t_{0}^{2}
\end{aligned}
$$

Now for $\sigma>0$ :

$$
\begin{aligned}
(A & -B)^{2}-\delta(A-\alpha B) A-\tau A^{2} \\
& =(A-\alpha B)^{2}+2(\alpha-1)(A-\alpha B) B+(\alpha-1)^{2} B^{2}-\delta(A-\alpha B) A-\tau A^{2} \\
& =(1-\sigma)(A-\alpha B)^{2}+(\sigma-\delta-\tau) A^{2}+[-2 \sigma \alpha+2(\alpha-1)+\delta \alpha] A B \\
& +\left(\sigma \alpha^{2}+1-\alpha^{2}\right) B^{2}
\end{aligned}
$$

First choose $\sigma$ such that $\sigma \alpha^{2}+1-\alpha^{2}=0$, that is:

$$
\sigma=\frac{\alpha^{2}-1}{\alpha^{2}} \text {. }
$$

Then $0<\sigma<1$. Next choose $\delta$ so that $-2 \sigma \alpha+2(\alpha-1)+\delta \alpha=0$, that is:

$$
\delta=\frac{2}{\alpha}(\sigma \alpha-\alpha+1)=\frac{2}{\alpha}\left(\frac{\alpha^{2}-1}{\alpha}-\alpha+1\right)=\frac{2(\alpha-1)}{\alpha^{2}}>0 .
$$

Then

$$
\eta-\delta=\frac{(\alpha-1)^{2}}{\alpha^{2}}>0
$$


Then we can choose $\tau=\frac{1}{2}(\sigma-\delta)$. Then

$$
(A-B)^{2}-\delta(A-\alpha B) A-\tau A \geq(1-\sigma)(A-\alpha B)^{2} .
$$

Note that $\sigma, \delta$ and $\tau$ depend only on $\alpha>1$. Put this back to (4.4), we have

$$
\begin{aligned}
0 \geq \phi F & {\left[-C_{8} t_{0}\left(1+\delta^{-1}\right)\left(R^{-1}+R^{-2}\right)-1\right]+\frac{2 t_{0}^{2}}{n+\epsilon}\left[(1-\sigma)(A-\alpha B)^{2}\right] } \\
& -C_{10}\left(1+\tau^{-1}\right) t_{0}^{2} \\
=\phi F & {\left[-C_{8} t_{0}\left(1+\delta^{-1}\right)\left(R^{-1}+R^{-2}\right)-1\right]+\frac{2}{n+\epsilon} \alpha^{-2}(\phi F)^{2} } \\
& +\frac{2 t_{0}^{2}}{n+\epsilon}\left[(A-B)^{2}-\delta(A-\alpha B) A-\tau A^{2}\right]-C_{10}\left(1+\tau^{-1}\right) t_{0}^{2}
\end{aligned}
$$

So

$$
\phi F \leq C_{11} t_{0}\left(R^{-2}+R^{-1}+1\right)+\frac{n+\epsilon}{2} \alpha^{2}
$$

on $M \times[0, T]$. Since $t_{0} \leq T$, we see that if $r(x) \leq \frac{1}{2} C_{2} R$, then

$$
F(x, T) \leq C_{11} T\left(R^{-2}+R^{-1}+1\right)+\frac{n+\epsilon}{2} \alpha^{2} .
$$

Since we can take any $t \in(0, T]$ to be our $T$, the result will follow by letting $R \rightarrow \infty$.

Corollary 4.1. Same assumptions as in the lemma, the following local version of gradient estimate is true:

$$
\begin{aligned}
\sup _{B_{p}\left(C_{2} R / 2\right)} & \left(\frac{|\nabla u|^{2}}{u^{2}}-\alpha \frac{u_{t}}{u}-\alpha q\right) \\
& \leq \frac{(n+\epsilon) \alpha^{2}}{2 t}+C_{11}\left(R^{-2}+R^{-1}+1\right)
\end{aligned}
$$

where the constants $C_{i}$ are as in the proof of the lemma.

Remark 4.1. Note that the constants in the local gradient estimates depend only on local data and the local behavior of the function $\rho$

Corollary 4.2. Let the $u$ be a positive solution of equation (2.2). Then, for any $\alpha>1$ and $\epsilon>0$, there are $C_{1}>0$ depending on $T$ and the upper bound of $|h|$, and $C_{2}>0$ depending on $\alpha, \epsilon, n, T$, the upper bounds of $|h|,\left|\nabla^{t} h\right|,|q|,\left|\nabla^{t} q\right|,\left|\Delta^{t} q\right|,\left|R c^{t}\right|$ and the curvature bound of the initial metric, such that

$$
u\left(x_{1}, t_{1}\right) \leq u\left(x_{2}, t_{2}\right)\left(\frac{t_{2}}{t_{1}}\right)^{\frac{(n+\epsilon) \alpha}{2}} \exp \left(\frac{C_{1} \alpha r^{2}\left(x_{1}, x_{2}\right)}{t_{2}-t_{1}}+C_{2}\left(t_{2}-t_{1}\right)\right) .
$$

for any $x_{1}, x_{2} \in M$ and $0<t_{1}<t_{2} \leq T$. 
Proof. Let $\left(x_{1}, t_{1}\right)$ and $\left(x_{2}, t_{2}\right)$ be two points in $M \times(0, T]$ with $t_{1}<t_{2}$. Let $\gamma(s)$ be a minimal geodesic joining $x_{1}$ to $x_{2}$ with respect to the initial metric. Let $l=r\left(x_{1}, x_{2}\right)$. Let $t(s)$ be an affine function such that $t(0)=t_{1}$ and $t(l)=t_{2}$. Then

$$
\begin{aligned}
\log \frac{u\left(x_{2}, t_{2}\right)}{u\left(x_{1}, t_{1}\right)} & =\int_{0}^{l} \frac{d}{d s} \log u(\gamma(s), t(s)) d s \\
& =\int_{0}^{l} \frac{\left\langle\nabla^{t} u, \gamma^{\prime}\right\rangle}{u}+t^{\prime} \frac{u_{t}}{u} d s \\
& \geq \int_{0}^{l}-C_{1} \frac{\left|\nabla^{t} u\right|}{u}+t^{\prime}\left(\frac{1}{\alpha} \frac{\left|\nabla^{t} u\right|^{2}}{u^{2}}-\frac{C_{2}}{\alpha}-\frac{(n+\epsilon) \alpha}{2 t(s)}-q\right) d s \\
& \geq-\frac{\alpha C_{1} l^{2}}{4\left(t_{2}-t_{1}\right)}-C_{3}\left(t_{2}-t_{1}\right)-\frac{n+\epsilon}{2} \log \frac{t_{2}}{t_{1}} .
\end{aligned}
$$

where $C_{1}$ depends only on the upper bound of $|h|$ and $T, C_{3}$ depends only on $\alpha, \epsilon, n, T$, the upper bounds of $|h|,\left|\nabla^{t} h\right|,|q|,\left|\nabla^{t} q\right|,\left|\Delta^{t} q\right|,\left|R c^{t}\right|$ and the bound of the sectional curvature of the initial metric. This completes the proof of the Corollary.

Corollary 4.3. Let $u$ be a positive solution of equation (2.2). Then there is a positive constant $C$ depending only on $n, T$, the upper bounds of $|h|,\left|\nabla^{t} h\right|,\left|\nabla^{t} q\right|,\left|\Delta^{t} q\right|,\left|R c^{t}\right|$ and the curvature bound of $g(0)$, such that for any $x \in M$ and $0<s<t \leq T$,

$$
u(x, s) \leq \frac{C}{V_{x}(\sqrt{t-s})}\left(\frac{t}{s}\right)^{n+1} \int_{B_{x}(\sqrt{t-s})} u(y, t) d V(y) .
$$

Proof. By Corollary 4.2,

$$
u(x, s) \leq C\left(\frac{t}{s}\right)^{n+1} u(y, t)
$$

for any $y \in B_{x}(\sqrt{t-s})$, by choosing $\epsilon=1, \alpha=2$, where $C$ depends on $n, T$, the upper bounds of $|h|,\left|\nabla^{t} h\right|,\left|\nabla^{t} q\right|,\left|\Delta^{t} q\right|,\left|R c^{t}\right|$ and the curvature bound of $g(0)$. Integrating on the both sides with respect to $d V(y)$ on $B_{x}(\sqrt{t-s})$, the result follows.

Remark 4.2. Since $g(t)$ is uniformly equivalent to $g(0)$, by volume comparison, we can see that in the corollary, the geodesic ball and its volume can be chosen with respect to any $g(t)$, perhaps with a different constant. 


\section{UPPER AND LOWER ESTIMATES OF THE FUNDAMENTAL SOLUTIONS}

In the following, we will apply the last three sections to get upper and lower estimates for the fundamental solutions of the equation (2.2). We always assume (A1)-(A3) in $\S 2$ are true.

Let $\mathcal{Z}(x, t ; y, s), 0 \leq s<t \leq T$ be the fundamental solution of equation (2.2):

$$
\frac{\partial}{\partial t} u-\Delta^{t} u+q u=0
$$

That is to say:

$$
\left\{\begin{array}{l}
\frac{\partial}{\partial t} \mathcal{Z}(x, t ; y, s)-\Delta_{x}^{t} \mathcal{Z}(x, t ; y, s)+q(x) \mathcal{Z}(x, t ; y, s)=0 \\
\lim _{t \rightarrow s} \mathcal{Z}(x, t ; y, s)=\delta_{y} .
\end{array}\right.
$$

The fundamental solution exists and is positive, see for example [12].

Then $\mathcal{Z}(x, t ; y, s)$ is the fundamental solution of the conjugate equation. That is:

$$
\left\{\begin{array}{l}
-\frac{\partial}{\partial s} \mathcal{Z}(x, t ; y, s)-\Delta_{y}^{s} \mathcal{Z}(x, t ; y, s)+(q(y)-H(y)) \mathcal{Z}(x, t ; y, s)=0 \\
\lim _{s \rightarrow t} \mathcal{Z}(x, t ; y, s)=\delta_{x} .
\end{array}\right.
$$

The fundamental solution $\mathcal{Z}(x, t ; y, s)$ can be obtained as follows.

Let $\Omega_{1} \subset \subset \Omega_{2} \subset \subset \cdots \subset \subset M$ be an exhaustion of relatively compact domains with smooth boundary in $M$. Let $\mathcal{Z}_{k}(x, t ; y, s)$ be the corresponding fundamental solution on $\Omega_{k}$ with zero Dirichlet boundary condition. Then $\mathcal{Z}_{k}$ is an increasing sequence by maximum principle and $\mathcal{Z}$ is the limit of $\mathcal{Z}_{k}$ as $k \rightarrow \infty$. Moreover, we have

$$
\mathcal{Z}_{k}(\cdot, \cdot ; y, s) \rightarrow \mathcal{Z}(\cdot, \cdot ; y, s)
$$

uniformly on any compact subset of $M \times(s, T]$ up to any derivatives, and

$$
\mathcal{Z}_{k}(x, t ; \cdot, \cdot) \rightarrow \mathcal{Z}(x, t ; \cdot, \cdot)
$$

uniformly on any compact subset of $M \times[0, t)$ up to any derivatives.

Lemma 5.1. There is a positive constant $C$ depending only on $T$ and the upper bounds of $|q|$ and $|H|$, such that

$$
\int_{M} \mathcal{Z}(x, t ; y, s) d V_{t}(x) \leq C
$$

for any $0<s<t \leq T$. Moreover, if $q=H$, then

$$
\int_{M} \mathcal{Z}(x, t ; y, s) d V_{t}(x)=1
$$

for any $0<s<t \leq T$. 
Proof. With the above notations, let

$$
I_{k}(t)=\int_{\Omega_{k}} \mathcal{Z}_{k}(x, t ; y, s) d V_{t}(x) .
$$

Then

$$
\begin{aligned}
& \frac{d}{d t} I_{k}(t) \\
= & \frac{d}{d t} \int_{\Omega_{k}} \mathcal{Z}_{k}(x, t ; y, s) d V_{t}(x) \\
= & \int_{\Omega_{k}}\left(\Delta_{x}^{t} \mathcal{Z}_{k}-q \mathcal{Z}_{k}\right) d V_{t}(x)+\int_{\Omega_{k}} H \mathcal{Z}_{k} d V_{t}(x) \\
= & \int_{\Omega_{k}}(H-q) \mathcal{Z}_{k} d V_{t}(x)+\int_{\partial \Omega_{k}} \frac{\partial Z_{k}}{\partial \vec{n}_{t}} d S_{t}(x) \\
\leq & \int_{\Omega_{k}}(H-q) \mathcal{Z}_{k} d V_{t}(x) \\
\leq & C_{1} I_{k}(t)
\end{aligned}
$$

since that $\mathcal{Z}_{k} \geq 0$ on $\Omega_{k} \times(s, T]$ and it is 0 on $\partial \Omega_{k} \times(s, T]$, where $C_{1}$ depends on the uniform upper bounds of $|q|$ and $|H|$. So

$$
\frac{d}{d t} \log I_{k}(t) \leq C_{1}
$$

Note that $I_{k}(s)=1$. Hence

$$
I_{k}(t) \leq e^{C_{1}(t-s)} \leq e^{C_{1} T} .
$$

By letting $k \rightarrow \infty$, we get the first inequality (5.3).

Suppose $q=H$. Let $\phi=\eta(\rho / R)$ be the same as in the proof of Lemma 4.1. For any $t_{1}, t_{2}$ with $s<t_{1}<t_{2} \leq T$, we have

$$
\begin{aligned}
& \left|\int_{M} \phi \mathcal{Z} d V_{t_{2}}(x)-\int_{M} \phi \mathcal{Z} d V_{t_{1}}(x)\right| \\
= & \left|\int_{t_{1}}^{t_{2}} \int_{M} \phi\left(Z_{t}+H \mathcal{Z}\right) d V_{t}(x) d t\right| \\
= & \left|\int_{t_{1}}^{t_{2}} \int_{M} \phi \Delta^{t} \mathcal{Z} d V_{t}(x) d t\right| \\
= & \left|\int_{t_{1}}^{t_{2}} \int_{M} \mathcal{Z} \Delta^{t} \phi d V_{t}(x) d t\right| \\
\leq & \frac{C_{2}\left(t_{2}-t_{1}\right)}{R} \cdot \max _{t_{1} \leq t \leq t_{2}} \int_{M} \mathcal{Z} d V_{t} \\
\leq & \frac{C_{3}}{R}
\end{aligned}
$$


where $C_{2}, C_{3}$ are independent of $R$ and (5.3) has been used. Let $R \rightarrow$ $\infty$, we get

$$
\int_{M} \mathcal{Z} d V_{t_{2}}(x)=\int_{M} \mathcal{Z} d V_{t_{1}}(x)
$$

for any $s<t_{1}<t_{2} \leq T$. Note that

$$
\lim _{t \rightarrow s^{+}} \int_{M} \mathcal{Z} d V_{t}(x)=1 \text {. }
$$

The result follows.

Corollary 5.1. There is a positive constant $C$ depending on $T$ and the upper bounds of $|q|$ and $|H|$, such that

$$
\int_{M} \mathcal{Z}(x, t ; y, s) d V_{s}(y) \leq C
$$

for any $s \in[0, t)$.

Proof. Since $\mathcal{Z}(x, t ; y, s)$ is also the fundamental solution of the conjugate equation, the proof is similar to the proof of Lemma 5.1 .

Lemma 5.2. There is a positive constant $C$ depending only on $T, n$, the lower bound of the Ricci curvature of the initial metric and the upper bounds of $|q|$ and $|h|$, such that

$$
\begin{aligned}
& \mathcal{Z}(x, t ; y, s) \leq \frac{C}{V_{x}(\sqrt{t-s})} \text { and } \\
& \mathcal{Z}(x, t ; y, s) \leq \frac{C}{V_{y}(\sqrt{t-s})} .
\end{aligned}
$$

Proof. Apply the mean value inequality Lemma 3.1 to

$$
\begin{aligned}
u(y, s)=\mathcal{Z}(x, t ; y, t-s) \text { with } r=\frac{\sqrt{s}}{2}, \text { we get } \\
\mathcal{Z}(x, t ; y, t-s)=u(y, s) \\
\leq \frac{C_{1} e^{A_{1} s+B_{1} r}}{r^{2} V_{y}(r)} \int_{0}^{s} \int_{M} u d V_{t} d s \\
\leq \frac{C_{2} e^{A_{1} s+B_{2} \sqrt{s}}}{V_{y}(\sqrt{s} / 2)} \\
\leq \frac{C_{3} e^{A_{1} s+B_{3} \sqrt{s}}}{V_{y}(\sqrt{s})} \\
\leq \frac{C_{3} e^{A_{1} T+B_{3} \sqrt{T}}}{V_{y}(\sqrt{s})}
\end{aligned}
$$

where in the last but second inequality we have used volume comparison. Here $C_{1}, C_{2}, C_{3}$ depend only on $n, T$, and the upper bounds of 
$q$ and $|h|, A_{1}$ depends only the upper bounds of $|q|$ and $|H|$, and $B_{1}$ depends only on $n$ and the lower bound of the Ricci curvature of $g(0)$. So, we get the second inequality in the lemma.

Applying similar method to $u(x, t)=\mathcal{Z}(x, t+s ; y, s)$ with $r=\frac{\sqrt{s}}{2}$ will get the first inequality.

Lemma 5.3. There are some positive constants $C$ and $D$ with $C$ depending only on $T, n$, the lower bound of the Ricci curvature of the initial metric and the upper bounds of $|q|$ and $|h|$, and $D$ depending only on $T$ and the upper bound of $|h|$, such that for $0 \leq s<t \leq T$,

$$
\begin{gathered}
\int_{M} \mathcal{Z}^{2}(x, t ; y, s) e^{\frac{r^{2}(x, y)}{D(t-s)}} d V_{t}(x) \leq \frac{C}{V_{y}(\sqrt{t-s})} \text { and } \\
\int_{M} \mathcal{Z}^{2}(x, t ; y, s) e^{\frac{r^{2}(x, y)}{D(t-s)}} d V_{s}(y) \leq \frac{C}{V_{x}(\sqrt{t-s})} .
\end{gathered}
$$

Proof. We just prove the first inequality. The proof of the second one is similar.

By Lemma 5.2 and the fact that $\mathcal{Z}_{k}$ increasing to $\mathcal{Z}$,

$$
\begin{aligned}
\int_{\Omega_{k}} \mathcal{Z}_{k}^{2}(x, t ; y, s) d V_{t}(x) & \leq \frac{C_{1}}{V_{y}(\sqrt{t-s})} \int_{\Omega_{k}} \mathcal{Z}_{k}(x, t ; y, s) d V_{t}(x) \\
& \leq \frac{C_{2}}{V_{y}(\sqrt{t-s})}
\end{aligned}
$$

for $0 \leq s<t \leq T$, where $C_{1}$ and $C_{2}$ depends on $T, n$, the lower bound of the Ricci curvature of the initial metric and the uniformly upper bounds of $|q|$ and $|h|$. Now fix $t>s$ and consider the function $u(x, \tau)=Z_{k}(x, \tau+s ; y, s), 0<\tau \leq t-s$.

Let

$$
f(\tau)=V_{y}(\sqrt{\tau})
$$

Then for $0<\tau_{1}<\tau_{2} \leq T$,

$$
\frac{f\left(\tau_{1}\right)}{f\left(\tau_{1} / 4\right)}=\frac{V_{y}\left(\sqrt{\tau_{1}}\right)}{V_{y}\left(\sqrt{\tau_{1}} / 2\right)} \leq \frac{V_{k}\left(\sqrt{\tau_{1}}\right)}{V_{k}\left(\sqrt{\tau_{1}} / 2\right)} \leq \frac{V_{k}(\sqrt{T})}{V_{k}(\sqrt{T} / 2)} \leq A \frac{f\left(\tau_{2}\right)}{f\left(\tau_{2} / 4\right)}
$$

where $V_{k}(r)$ denotes the volume of the ball of radius $r$ in the space form with Ricci curvature $-k$ ( $-k$ is the lower bound of the Ricci curvature of the initial metric) and $A=\frac{V_{k}(\sqrt{T})}{V_{k}(\sqrt{T} / 2)}$. So, $f$ is regular with the constants $A$ and $\gamma=4$. By Lemma 2.1,

$$
\int_{\Omega_{k}} \mathcal{Z}_{k}^{2}(x, t ; y, s) e^{\frac{r^{2}(x, y)}{D(t-s)}} d V_{t}(x) \leq \frac{C}{V_{y}(\sqrt{t-s})}
$$


where $D$ depends on $T$ and the uniformly upper bound of $|h|$, and $C$ depends on $T, n$, the lower bound of the Ricci curvature of the initial metric and the uniformly upper bounds of $|q|$ and $|h|$.

By taking limit, we get the first inequality.

Theorem 5.1. There exist positive constants $C$ and $D$ with $C$ depending only on $T, n$, the lower bound of the Ricci curvature of the initial metric and the upper bounds of $|q|$ and $|h|$, and $D$ depending only on $T$ and the upper bound of $|h|$, such that for $0 \leq s<t \leq T$,

$$
\mathcal{Z}(x, t ; y, s) \leq \frac{C}{V_{x}^{\frac{1}{2}}(\sqrt{t-s}) V_{y}^{\frac{1}{2}}(\sqrt{t-s})} \times e^{-\frac{r^{2}(x, y)}{D(t-s)}} .
$$

Proof. By the triangle inequality, we have

$$
r^{2}(x, \zeta)+r^{2}(\zeta, y)-\frac{r^{2}(x, y)}{2} \geq 0
$$

Let $D$ be as in Lemma 5.3 and let $\tau=(s+t) / 2$. Then by the semigroup property and Lemma 5.3 ,

$$
\begin{aligned}
& \mathcal{Z}(x, t ; y, s) \\
= & \int_{M} \mathcal{Z}(x, t ; \zeta, \tau) \mathcal{Z}(\zeta, \tau ; y, s) d V_{\tau}(\zeta) \\
\leq & \int_{M} \mathcal{Z}(x, t ; \zeta, \tau) \mathcal{Z}(\zeta, \tau ; y, s) e^{\frac{r^{2}(x, \zeta)}{2 D(t-s)}+\frac{r^{2}(\zeta, y)}{2 D(t-s)}-\frac{r^{2}(x, y)}{4 D(t-s)}} d V_{\tau}(\zeta) \\
\leq & e^{-\frac{r^{2}(x, y)}{4 D(t-s)}}\left(\int_{M} \mathcal{Z}^{2}(x, t ; \zeta, \tau) e^{\frac{r^{2}(x, \zeta)}{D(t-s)}} d V_{\tau}(\zeta)\right)^{1 / 2} \\
& \times\left(\int_{M} \mathcal{Z}^{2}(\zeta, \tau ; y, s) e^{\frac{r^{2}(\zeta, y)}{D(t-s)}} d V_{\tau}(\zeta)\right)^{1 / 2} \\
\leq & \frac{C}{V_{x}^{\frac{1}{2}}(\sqrt{t-s}) V_{y}^{\frac{1}{2}}(\sqrt{t-s})} \times e^{-\frac{r^{2}(x, y)}{4 D(t-s)}}
\end{aligned}
$$

Corollary 5.2. There exist positive constants $C$ and $D$ with $C$ depending only $T, n$, the lower bound of the Ricci curvature of the initial metric and the upper bounds of $|q|$ and $|h|$, and $D$ depending only on $T$ and the upper bound of $|h|$, such that

$$
\begin{gathered}
\mathcal{Z}(x, t ; y, s) \leq \frac{C}{V_{x}(\sqrt{t-s})} e^{-\frac{r^{2}(x, y)}{D(t-s)}} \text { and } \\
\mathcal{Z}(x, t ; y, s) \leq \frac{C}{V_{y}(\sqrt{t-s})} e^{-\frac{r^{2}(x, y)}{D(t-s)}}
\end{gathered}
$$


for any $0<s<t<T$.

Proof. We just prove the first inequality, the proof of the other one is similar.

By Proposition 5.1 and volume comparison,

$$
\begin{aligned}
& \mathcal{Z}(x, t ; y, s) \\
\leq & \frac{C_{1}}{V_{x}^{\frac{1}{2}}(\sqrt{t-s}) V_{y}^{\frac{1}{2}}(\sqrt{t-s})} \times e^{-\frac{r^{2}(x, y)}{D_{1}(t-s)}} \\
\leq & \frac{C_{1}}{V_{x}^{\frac{1}{2}}(\sqrt{t-s}) V_{y}^{\frac{1}{2}}(\sqrt{t-s})} \times e^{-\frac{r^{2}(x, y)}{D_{1}(t-s)}} \times \frac{V_{y}^{\frac{1}{2}}(r(x, y)+\sqrt{t-s})}{V_{x}^{\frac{1}{2}}(\sqrt{t-s})} \\
\leq & \frac{C_{1}}{V_{x}(\sqrt{t-s})} \times e^{-\frac{r^{2}(x, y)}{D_{1}(t-s)}} \times \frac{V_{y}(r(x, y)+\sqrt{t-s})}{V_{y}(\sqrt{t-s})} \\
\leq & \frac{C_{1}}{V_{x}(\sqrt{t-s})} e^{-\frac{r^{2}(x, y)}{D_{1}(t-s)}+(n-1) \sqrt{k}(r(x, y)+\sqrt{t-s})+n \frac{r(x, y)}{\sqrt{t-s}}} \\
\leq & \frac{C_{1}}{V_{x}(\sqrt{t-s})} e^{-\frac{r^{2}(x, y)}{D_{1}(t-s)}+C_{2} \frac{r(x, y)}{\sqrt{t-s}}} .
\end{aligned}
$$

So, when $\frac{r(x, y)}{\sqrt{t-s}} \geq 2 C_{2} D_{1}$, we have

$$
\mathcal{Z}(x, t ; y, s) \leq \frac{C_{1}}{V_{x}(\sqrt{t-s})} e^{-\frac{r^{2}(x, y)}{2 D_{1}(t-s)}}
$$

For those that $\frac{r(x, y)}{\sqrt{t-s}} \leq 2 C_{2} D_{1}$, by Lemma $[5.2$, we have

$$
\begin{aligned}
& \mathcal{Z}(x, t ; y, s) \\
\leq & \frac{C_{3}}{V_{x}(\sqrt{t-s})} \\
= & \frac{C_{3}}{V_{x}(\sqrt{t-s})} \times e^{-\frac{r^{2}(x, y)}{2 D_{1}(t-s)}} \times e^{\frac{r^{2}(x, y)}{2 D_{1}(t-s)}} \\
\leq & \frac{C_{4}}{V_{x}(\sqrt{t-s})} \times e^{-\frac{r^{2}(x, y)}{2 D_{1}(t-s)}} .
\end{aligned}
$$

This complete the proof the first inequality.

Next we want to obtain lower estimates of the fundamental solution. We will proceed as in [8]. 
Lemma 5.4. There is a positive constant $c$ depending only on $T$ and the upper bounds of $|q|$ and $|H|$, such that

$$
\begin{aligned}
& \int_{M} \mathcal{Z}(x, t ; y, s) d V_{t}(x) \geq c \text { and } \\
& \int_{M} \mathcal{Z}(x, t ; y, s) d V_{s}(y) \geq c
\end{aligned}
$$

for any $0<s<t<T$.

Proof. We just prove the first inequality, and the proof of the second one is similar. Let $\phi=\eta(\rho / R)$ be the same function as in the proof of Lemma 4.1. Then for any $t_{1}<t_{2}$ in $(s, T)$,

$$
\begin{aligned}
& \frac{d}{d t} \int_{M} \phi \mathcal{Z} d V_{t} \\
= & \int_{M} \phi \Delta^{t} \mathcal{Z}+(H-q) \phi \mathcal{Z} d V_{t} \\
\geq & \int_{M} \mathcal{Z} \Delta^{t} \phi d V_{t} d t-C_{1} \int_{M} \phi \mathcal{Z} d V_{t} \\
\geq & -\frac{C_{2}}{R}-C_{1} \int_{M} \phi \mathcal{Z} d V_{t}
\end{aligned}
$$

where $C_{1}$ depends on the uniformly upper bounds of $|q|$ and $|H|, C_{2}$ is independent of $R$ and Lemma 5.1 has been used in the last inequality. So

$$
\begin{gathered}
\frac{d}{d t}\left(e^{C_{1}(t-s)} \int_{M} \phi \mathcal{Z} d V_{t}\right) \geq-\frac{C_{2} e^{C_{1}(t-s)}}{R} \text { and } \\
\int_{M} \phi \mathcal{Z} d V_{t} \geq e^{-C_{1}(t-s)}\left\{e^{C_{1}\left(t_{1}-s\right)} \int_{M} \phi \mathcal{Z} d V_{t_{1}}-\frac{C_{2}\left(1-e^{C_{1}(t-s)}\right)}{C_{1} R}\right\}
\end{gathered}
$$

for any $T>t>t_{1}>s$. Let $R \rightarrow \infty$ and $t_{1} \rightarrow s^{+}$, we get

$$
\int_{M} \mathcal{Z} d V_{t} \geq e^{-C_{1}(t-s)} \geq e^{-C_{1} T}
$$

for any $t \in(s, T)$.

Lemma 5.5. Let $c$ be the constant in Lemma 5.4. Then, there is a constant $A>1$ depending only on $n, T$, the lower bound of the Ricci curvature of the initial metric and the upper bounds of $|q|$ and $|h|$, such that

$$
\begin{gathered}
\int_{B_{y}(A \sqrt{t-s})} \mathcal{Z}(x, t ; y, s) d V_{t}(x) \geq \frac{c}{2} \text { and } \\
\int_{B_{x}(A \sqrt{t-s})} \mathcal{Z}(x, t ; y, s) d V_{s}(y) \geq \frac{c}{2}
\end{gathered}
$$


for any $0<s<t<T$.

Proof. we just prove that first inequality, the proof of second one is similar.

By the second inequality of Corollary 5.2

$$
\begin{aligned}
& \int_{M \backslash B_{y}(A \sqrt{t-s})} \mathcal{Z}(x, t ; y, s) d V_{t}(x) \\
\leq & \frac{C_{1}}{V_{y}(\sqrt{t-s})} \int_{M \backslash B_{y}(A \sqrt{t-s})} e^{-\frac{r^{2}(x, y)}{D(t-s)}} d V(x) \\
= & \frac{C_{1}}{V_{y}(\sqrt{t-s})} \int_{A \sqrt{t-s}}^{\infty} e^{-\frac{r^{2}}{D(t-s)}} d V_{y}(r) \\
\leq & C_{1} \int_{A \sqrt{t-s}}^{\infty} \frac{V_{y}(r)}{V_{y}(\sqrt{t-s})} \times e^{-\frac{r^{2}}{D(t-s)}} d\left(\frac{2 r}{D(t-s)}\right) \\
\leq & \frac{C_{1}}{D} \int_{A \sqrt{t-s}}^{\infty}\left(\frac{r}{\sqrt{t-s}}\right)^{n} e^{-\frac{r^{2}}{D(t-s)}+C_{2} \frac{r}{\sqrt{t-s}}} d\left(\frac{r^{2}}{t-s}\right)
\end{aligned}
$$

where $C_{2}$ depends on $n, T$ and the lower bound of the Ricci curvature of the initial metric.

If we first require that $A \geq 2 C_{2} D$, then

$$
\begin{aligned}
& \int_{M \backslash B_{y}(A \sqrt{t-s})} \mathcal{Z}(x, t ; y, s) d V_{t}(x) \\
\leq & \frac{C_{1}}{D} \int_{A \sqrt{t-s}}^{\infty}\left(\frac{r}{\sqrt{t-s}}\right)^{n} e^{-\frac{r^{2}}{2 D(t-s)}} d\left(\frac{r^{2}}{t-s}\right) \\
= & 2 C_{3} \int_{2 D A^{2}}^{\infty} x^{\frac{n}{2}} e^{-x^{2}} d x \\
\leq & \frac{c}{2}
\end{aligned}
$$

when $A$ is large enough depending only on $n, T$, the lower bound of the Ricci curvature of the initial metric and the upper bounds of $|q|$ and $|h|$. This complete the proof of the first inequality.

Lemma 5.6. There is a constant $c>0$ depending on $n, T$ and the uniformly upper bounds of $|h|,\left|\nabla^{t} h\right|,|q|,\left|\nabla^{t} q\right|,\left|\Delta^{t} q\right|,\left|R c^{t}\right|$, such that

$$
\begin{gathered}
\mathcal{Z}(x, t ; x, s) \geq \frac{c}{V_{x}(\sqrt{t-s})} \text { and } \\
\mathcal{Z}(x, t ; y, s) \geq \frac{c}{V_{y}(\sqrt{t-s})}
\end{gathered}
$$

for any $x, y$ and $0<s<t<T$ 
Proof. We just prove the first inequality, the proof of the second one is similar.

Let $\tau=\frac{t-s}{2}$. Then, by Corollary 4.2 ,

$$
c_{1} e^{-\frac{r^{2}(x, y)}{c_{2} \tau}} \mathcal{Z}(y, t-\tau ; x, s) \leq \mathcal{Z}(x, t ; x, s)
$$

where $c_{1}>0$ depends only on $n, T$ and the upper bounds of $|h|,\left|\nabla^{t} h\right|,|q|$, $\left|\nabla^{t} q\right|,\left|\Delta^{t} q\right|,\left|R c^{t}\right|$, and $c_{2}>0$ depends only on $T$ and the upper bound of $|h|$. This implies that

$c_{1} \int_{B_{x}(A \sqrt{\tau})} e^{-\frac{r^{2}(x, y)}{c_{2} \tau}} \mathcal{Z}(y, t-\tau ; x, s) d V(y) \leq \int_{B_{x}(A \sqrt{t})} \mathcal{Z}(x, t ; x, s) d V(y)$.

where $A$ is the same as in Lemma 5.5. By Lemma 5.5.

$$
\mathcal{Z}(x, t ; x, s) \geq \frac{c_{3}}{V_{x}(A \sqrt{\tau})} \geq \frac{c_{4}}{V_{x}(\sqrt{t-s})}
$$

with $c_{3}$ and $c_{4}$ depending on $n, T$ and the upper bounds of $|h|,\left|\nabla^{t} h\right|,|q|$, $\left|\nabla^{t} q\right|,\left|\Delta^{t} q\right|,\left|R c^{t}\right|$.

This completes the proof of the first inequality.

Proposition 5.1. There exist positive constants $c$ and $d$ with $c$ depending on $n, T$ and the upper bounds of $|h|,\left|\nabla^{t} h\right|,|q|,\left|\nabla^{t} q\right|,\left|\Delta^{t} q\right|,\left|R c^{t}\right|$, and $d$ depending only on $T$ and the upper bound of $|h|$, such that

$$
\begin{gathered}
\mathcal{Z}(x, t ; y, s) \geq \frac{c}{V_{x}(\sqrt{t-s})} \times e^{-\frac{r^{2}(x, y)}{d(t-s)}} \text { and } \\
\mathcal{Z}(x, t ; y, s) \geq \frac{c}{V_{y}(\sqrt{t-s})} \times e^{-\frac{r^{2}(x, y)}{d(t-s)}} .
\end{gathered}
$$

for any $0<s<t<T$.

Proof. We just prove the second inequality, the proof of the first one is similar.

Let $\tau=\frac{t-s}{2}$. By Corollary 4.2 and Lemma 5.6,

$$
\begin{aligned}
& \mathcal{Z}(x, t ; y, s) \\
\geq & c_{1} \mathcal{Z}(y, t-\tau ; y, s) e^{-\frac{r^{2}(x, y)}{c_{2} \tau}} \\
\geq & \frac{c_{3}}{V_{y}(\sqrt{\tau})} e^{-\frac{r^{2}(x, y)}{c_{2} \tau}} \\
\geq & \frac{c_{4}}{V_{y}(\sqrt{t-s})} e^{-\frac{r^{2}(x, y)}{c_{2}(t-s)}} .
\end{aligned}
$$


Corollary 5.3. For the same positive constants $c$ and $d$ as in Proposition 5.1,

$$
\mathcal{Z}(x, t ; y, s) \geq \frac{c}{V_{x}^{\frac{1}{2}}(\sqrt{t-s}) V_{y}^{\frac{1}{2}}(\sqrt{t-s})} \times e^{-\frac{r^{2}(x, y)}{d(t-s)}}
$$

for any $0<s<t<T$.

Proof. Multiplying the two inequalities in Proposition 5.1 the result follows.

\section{More Gradient estimates}

In this section we want to obtain more gradient estimates, which are generalizations of the gradient estimates in [35] and [20] to complete noncompact manifolds. The estimates will be used in later sections.

Let $u>0$ be a solution of the equation:

$$
\Delta^{t} u-u_{t}=0
$$

on $M \times[0, T]$ corresponding to the Ricci flow

$$
\frac{\partial}{\partial t} g=-2 R i c
$$

or a solution of

$$
\Delta^{\tau} u-u_{\tau}-R u=0
$$

on $M \times[0, T]$ corresponding to the backward Ricci flow

$$
\frac{\partial}{\partial \tau} g=2 R i c
$$

where $R$ is the scalar curvature, $\tau=T-t$.

Let use make the following assumption:

(a1) $g(t)$ is complete and $\left|\nabla^{k} R m\right|$ are uniformly bounded on spacetime by $c_{k}$ for all $k$.

Lemma 6.1. Let $u>0$ be a solution of (6.3) or (6.1) such that $u \leq A$ for all $t$. There is a constant $C$ depending only on those constants $c_{k}$ for $k=0,1$ in the assumption (a1), $n, T$ and $A$, such that

$$
|\nabla u|(x, t) \leq C / t
$$

for all $x \in M \times(0, T]$.

Before we prove the lemma, let us modify a maximum principle in [9]. 
Lemma 6.2. Suppose $g(t)$ is a smooth family of complete metrics defined on $M, 0 \leq t \leq T$ with Ricci curvature bounded from below and $\left|\frac{\partial}{\partial t} g\right| \leq C$ on $M \times[0, T]$. Suppose $f(x, t)$ is a smooth function defined on $M \times[0, T]$ such that

$$
\Delta^{t} f-\frac{\partial}{\partial t} f \geq 0
$$

and

$$
\int_{0}^{T} \int_{M} \exp \left(-a r_{t}^{2}(o, x)\right) f^{2}(x, t) d V_{t}<\infty
$$

for some $a>0$. If $f(x, 0) \leq 0$ for all $x \in M$, then $f \leq 0$ on $M \times[0, T]$.

Proof. In [9], the condition (6.6) with $f$ being replaced by $|\nabla f|$ is assumed. From the their proof, it is easy to see that the result is still true if $f$ is replaced by $\left|\nabla f_{+}\right|$, where $f_{+}=\max \{f, 0\}$.

Observe that by (6.6), there exists $T_{i} \uparrow T$ such that

$$
\int_{M} \exp \left(-a r_{T_{i}}^{2}(o, x)\right) f^{2}\left(x, T_{i}\right) d V_{T_{i}}<\infty .
$$

Then one can obtain their condition by using (6.6) and (6.5) by a cutoff argument on $\left[0, T_{i}\right]$, perhaps with another $a_{i}>0$.

Proof of Lemma 6.1. In the following $C_{i}$ 's will denote constants depending only on the quantities mentioned in the lemma.

Let us prove the case that $u$ is a solution of $(\underline{6.3})$. The other case is similar. Note that we may consider the interval $[\epsilon, T]$ first, and then let $\epsilon \rightarrow 0$. Hence we may assume that $u$ is smooth up to $\tau=0$. For simplicity, we will write $\tau$ as $t$, and $\Delta$ instead of $\Delta^{t}$.

Let us compute the followings in normal coordinates at a point with respect to $g(t)$

$$
\begin{aligned}
\left(\Delta-\partial_{t}\right)|\nabla u|^{2} & =2 \sum_{i j} u_{i j}^{2}+2 \sum_{i}(\Delta u)_{i} u_{i}+4 \sum_{i j} R_{i j} u_{i} u_{j}-2 \sum_{i} u_{t i} u_{i} \\
& =2 \sum_{i j} u_{i j}^{2}+4 \sum_{i j} R_{i j} u_{i} u_{j}+2 \sum_{i}(R u)_{i} u_{i} \\
& \geq 2 \sum_{i j} u_{i j}^{2}-C_{1}\left(|\nabla u|^{2}+1\right) .
\end{aligned}
$$


Here and below, $C_{i}$ denotes constant depending only on $n, T$ and $c_{0}, c_{1}$. Let $f=\left(|\nabla u|^{2}+1\right)^{\frac{1}{2}}$. Then

$$
\begin{aligned}
4 f^{2}|\nabla f|^{2} & =\left|\nabla f^{2}\right|^{2} \\
& =4 \sum_{j}\left(\sum_{i} u_{i j} u_{i}\right)^{2} \\
& \leq 4 f^{2} \sum_{i j} u_{i j}^{2} . \\
2 f\left(\Delta-\partial_{t}\right) f & =\left(\Delta-\partial_{t}\right) f^{2}-2|\nabla f|^{2} \\
& \geq-C_{1} f^{2}
\end{aligned}
$$

and so

$$
\left(\Delta-\partial_{t}\right)(t f) \geq-C_{1} f
$$

$$
\left(\Delta-\partial_{t}\right)\left(\frac{1}{2} C_{1} u^{2}-C_{2} t-\frac{1}{2} C_{1} A^{2}\right)=C_{1}|\nabla u|^{2}+C_{1} R u^{2}+C_{2} \geq C_{1} f^{2} \geq C_{1} f
$$

where $C_{2}$ is chosen so that $C_{1} R u^{2}+C_{2}-C_{1} \geq 0$.

Hence

$$
\left(\Delta-\partial_{t}\right) Q \geq 0
$$

where

$$
Q=t f+\frac{2}{C_{1}} u^{2}-C_{2} t-\frac{1}{2} C_{1} A^{2} .
$$

Since $Q \leq 0$, at $t=0$, the result will follow from Lemma 6.2, provided that we can prove:

$$
\int_{0}^{T} \int_{M} \exp \left(-a^{2} r_{t}^{2}\right) Q^{2} d V_{t} d t<\infty
$$

for some $a>0$. Here $r_{t}(x)$ is the distance from a fixed point. Since the curvature is bounded, by volume comparison, it is sufficient to prove that

$$
\int_{0}^{T} \int_{M} \exp \left(-a^{2} r_{t}^{2}\right)|\nabla u|^{2} d V_{t} d t<\infty
$$

for some $a>0$. Here $r_{t}(x)$ is the distance from a fixed point in $g(t)$. Since we have

$$
\left(\Delta-\frac{\partial}{\partial t}\right)\left(\exp \left(-C_{3} t\right) u\right) \geq 0 \text {. }
$$

Using a cutoff argument and the fact that $u$ is bounded, it is easy to see that (6.13) is true. 
Lemma 6.3. With the same notations and assumptions as before, the following estimates are true:

Suppose $u>0$ is a solution of (6.3) or (6.1), and suppose $u \leq A$. Then

$$
t \frac{|\nabla u|^{2}}{u} \leq C\left[u \log \frac{A}{u}+u\right]
$$

for some constant $C$ depending only on $T$, $n$ and $c_{k}, 0 \leq k \leq 2$, in the assumption (a1).

Proof. We prove the case that $u$ is a solution of (6.3) and the other case can be proved similarly. As in [23], for some suitable positive constants $C_{1}, C_{2}, C_{3}$ such that if we let

$$
\Phi=\varphi \frac{|\nabla u|^{2}}{u}-\exp \left(C_{1} \tau\right) u \log \left(\frac{A}{u}\right)-C_{2} \tau u
$$

where $\varphi=\tau /\left(1+C_{3} \tau\right)$, then

$$
\left(\Delta^{t}-\frac{\partial}{\partial \tau}\right) \Phi \geq 0
$$

Here and below, $C_{i}$ 's will denote constants depending on quantities mentioned in the lemma. Again, we may consider $u$ being a solution in the interval $[\epsilon, T]$ for $\epsilon>0$ first, and $\tau=0$ corresponding to the original time $\epsilon$. Let $p \in M$ be a fixed point, then by Corollary 4.2

$$
u\left(p, \frac{\epsilon}{2}\right) \leq\left(C_{4}+c\right) u(x, \tau) \exp \left(\left(C_{4}+c\right) r^{2}(p, x)\right)
$$

for some $c$ depends only on quantities mentioned in the lemma and $\epsilon$, and $\epsilon \leq \tau \leq T$. By Lemma 6.1, and the fact that $u$ is bounded, we conclude that there is $a>0$ such that $\exp \left(-a r_{t}(p, x)^{2}\right) \Phi^{2}$ is integrable on $M \times[\epsilon, T]$ with respect to $d V_{\tau} d \tau$. Since $\Phi \leq 0$ initially, we can apply Lemma 6.2 to conclude that the lemma is true for bounded and positive solutions of (6.3).

\section{A Li-Yau-Hamilton type Differential inequality}

Let $\left(M^{n}, g(t)\right)$ be a solution of the Ricci flow:

$$
\frac{\partial g}{\partial t}=-2 R i c
$$

on $M \times[0, T]$ for some $T>0$. We always assume that $M^{n}$ is noncompact, $g(t)$ is complete and (a1) in the previous section is true. Let $\mathcal{Z}(x, t ; y, s)$ with $0 \leq s<t \leq T$ be the fundamental solution of

$$
\frac{\partial u}{\partial t}-\Delta^{t} u=0
$$


Let $p \in M$ be fixed and let $u(x, t)=\mathcal{Z}(p, T ; x, t)>0$. Then $u$ is a solution of the conjugate heat equation

$$
-\frac{\partial u}{\partial t}-\Delta^{t} u+\mathcal{R} u=0
$$

on $M \times[0, T]$, where $\mathcal{R}$ is the scalar curvature and $\Delta^{t}$ is the Laplacian with respect to $g(t)$. When there is no confusion, we simply denote $\Delta^{t}$ as $\Delta$. Let $v$ be defined by

$$
v=\left[\tau\left(2 \Delta f-|\nabla f|^{2}+R\right)+(f-n)\right] u
$$

where $f$ is defined by $u=e^{-f} /(4 \pi \tau)^{\frac{n}{2}}$ and $\tau=T-t$ (This notation is adopted throughout this section).

Let $h_{0} \geq 0$ be a smooth function with compact support and let $0<t_{0}<T$. Let $h(x, t)$ be the solution of $(7.2)$ on $M \times\left[t_{0}, T\right]$ with initial data $h\left(x, t_{0}\right)=h_{0}(x)$. We want to prove the following:

Theorem 7.1. With the above notations and assumption (a1), we have

(i) For any $t_{0}<t<T \cdot h v(\cdot, t) \in L^{1}(M, g(t))$,

(ii) For any $t_{0}<t_{1}<t_{2}<T$

$$
\int_{M} h v d V_{t_{1}} \leq \int_{M} h v d V_{t_{2}} .
$$

$$
\limsup _{t \rightarrow T^{-}} \int_{M} h v d V_{t} \leq 0 .
$$

Lemma 7.1. Theorem 7.1(i) is true.

Proof. For any $T>t>t_{0}$, by Corollary 5.2 and Lemma 6.3, there are $a, C_{1}>0$ such that for all $x \in M$

$$
\left(\frac{|\nabla u|^{2}}{u}+u+h+|\nabla h|\right)(x, t) \leq C_{1} \exp \left(-a r^{2}(x)\right) .
$$

Since the curvature is bounded and since $-f=\log u+\frac{n}{2} \log (4 \pi \tau)$, $|\nabla f|^{2} u h$, fuh, nuh, Ruh are all in $L^{1}$, where $\tau=T-t$. Moreover, since

$$
\Delta f=-\frac{\Delta u}{u}+|\nabla f|^{2}
$$

in order to prove the lemma, it is sufficient to prove that $h \Delta u$ is in $L^{1}$. By Lemma 4.1, we have for $T>t>0$,

$$
\frac{|\nabla u|^{2}}{u^{2}}-\alpha \frac{u_{\tau}}{u}-C_{2} \leq 0
$$


for some $C_{2}>0$. Hence

$$
\begin{aligned}
\int_{M}|h \Delta u| & =\int_{M}\left|u_{\tau}-R u\right| h \\
& \leq \alpha^{-1} \int_{M}\left(\alpha u_{\tau}-\frac{|\nabla u|^{2}}{u}+C_{2}\right) h+C_{3} \\
& =\int_{M} h \Delta u+C_{4} \\
& <C_{5}
\end{aligned}
$$

for some constants $C_{3}-C_{5}$, where have has used (7.5), integration by parts together with a cutoff argument. This completes the proof of the lemma.

Remark 7.1. From the proof, it is easy to see that for $0<\tau_{1}<\tau_{2}<T$, there is a constant $C$ so that

$$
\int_{M}(|v h|+|v|) d V_{t} \leq C
$$

for all $\tau_{1} \leq \tau \leq \tau_{2}$. Moreover, (7.5) is true for a constant $C$ for all $\tau_{1} \leq \tau \leq \tau_{2}$.

Lemma 7.2. Theorem 7.1](ii) is true.

Proof. By [26], we have

$$
\left(\frac{\partial}{\partial \tau}-\Delta+R\right) v=-2 \tau\left|R_{i j}+f_{i j}-\frac{1}{\tau} g_{i j}\right|^{2} u,
$$

where $\tau=T-t$. Let $\rho$ and $\phi$ be the functions defined in the proof of Lemma 4.1. Fix $0<t_{1}<t_{2}<T$, by Remark 7.1 and (7.5) for any $t_{1} \leq t \leq t_{2}$

$$
\begin{aligned}
\frac{d}{d \tau} \int_{M} \phi h v d V_{t} & =\int_{M} \phi\left(v_{\tau} h+v h_{\tau}+v h R\right) d V_{t} \\
& \leq \int_{M} \phi(h \Delta v-v \Delta h) d V_{t} \\
& =\int_{M}(v\langle\nabla \phi, \nabla h\rangle-h\langle\nabla \phi, \nabla v\rangle) d V_{t} \\
& =\int_{M}(2 v\langle\nabla \phi, \nabla h\rangle+h v \Delta \phi) d V_{t} \\
& \leq \frac{C}{R}
\end{aligned}
$$

for some $C>0$ for all $\tau_{1} \leq \tau \leq \tau_{2}$. By integrating from $\tau_{1}$ to $\tau_{2}$, and letting $R \rightarrow \infty$, the result follows. 
Next, we want to prove Theorem 7.1(iii). We need several lemmas.

Lemma 7.3. For any $\alpha>1$ and $\delta, \epsilon>0$, there is a constant $C(\alpha, \delta, \epsilon)$ which is independent of $t$ such that, if $\frac{T}{2}<t<T$, then

$$
(1-2 \alpha \delta) \int_{M} \frac{|\nabla u|^{2}}{u} h d V_{t} \leq C+\frac{(n+\epsilon) \alpha^{2}}{2 \tau} \int_{M} u h d V_{t}
$$

where $C$ is a constant independent of $t$ and $\tau=T-t$.

Proof. Let $\alpha$ and $\epsilon$ be given, then by Lemma 4.1,

$$
\frac{|\nabla u|^{2}}{u} \leq \alpha\left(u_{\tau}+R u\right)+C_{1}+\frac{(n+\epsilon) \alpha^{2}}{2 \tau}=\alpha \Delta u+C_{1}+\frac{(n+\epsilon) \alpha^{2}}{2 \tau} .
$$

Here and below, $C_{i}$ 's are positive constant which is independent of $\tau$. Let $\rho$ and $\phi$ be as in the proof of Lemma 4.1. Then

$$
\begin{aligned}
\int_{M} \phi^{2} \frac{|\nabla u|^{2}}{u} h d V_{t} \leq & \alpha \int_{M} \phi^{2} h \Delta u d V_{t}+C_{1} \int_{M} \phi^{2} u h d V_{t} \\
+ & \frac{(n+\epsilon) \alpha^{2}}{2 \tau} \int_{M} \phi^{2} u h d V_{t} \\
\leq & \alpha\left(-2 \int_{M} \phi h\langle\nabla u, \nabla \phi\rangle d V_{t}-\int_{M} \phi^{2}\langle\nabla u, \nabla h\rangle d V_{\tau}\right) \\
& +C_{2}+\frac{(n+\epsilon) \alpha^{2}}{2 \tau} \int_{M} u h d V_{t} \\
\leq & 2 \alpha \delta \int_{M} \phi^{2} \frac{|\nabla u|^{2}}{u} h d V_{t}+\frac{C_{3} \alpha}{\delta R^{2}} \int_{M} h u d V_{t} \\
& +\frac{\alpha}{\delta} \int_{M} \phi^{2} \frac{|\nabla h|^{2}}{h} u d V_{t}+C_{2}+\frac{(n+\epsilon) \alpha^{2}}{2 \tau} \int_{M} u h d V_{t}
\end{aligned}
$$

where $C_{i}$ 's do not depend on $\tau$. Note that $\int_{M} h u d V_{t}$ is bounded by a constant independent of $\tau \in(0, T / 2)$ because $h$ is bounded and

$$
\int_{M} u d V_{t}
$$

is bounded independent of $\tau$ by Lemma 5.1. Also, $|\nabla h|^{2} / h$ is bounded independent of $\tau \in(0, T / 2)$ by Lemma 6.3, Let $R \rightarrow \infty$, we have

$$
(1-2 \alpha \delta) \int_{M} \frac{|\nabla u|^{2}}{u} h d V_{t} \leq C_{1}(\alpha, \epsilon, \delta)+\frac{(n+\epsilon) \alpha^{2}}{2 \tau} \int_{M} u h d V_{t}
$$


Lemma 7.4. For any $\delta>0$,

$$
\int_{M}(-\Delta u) h d V_{t} \leq 2 \delta \int_{M} \frac{|\nabla u|^{2}}{u} h d V_{t}+C
$$

where $C$ is a constant independent of $t$, provided $0<\tau<\frac{T}{2}$.

Proof. Let $\phi$ be then same as before. As in the proof of Lemma [7.3,

$$
\int_{M} \phi^{2}(-\Delta u) h d V_{\tau} \leq 2 \delta \int_{M} \phi^{2} \frac{|\nabla u|^{2}}{u} h d V_{t}+\frac{C}{\delta R^{2}} \int_{M} h u d V_{t}+C
$$

where $C$ is independent of $\tau$. Since $h \Delta u$ is in $L^{1}(M, g(t))$ by the proof of Lemma 7.1, let $R \rightarrow \infty$, the result follows.

\section{Lemma 7.5.}

$$
\limsup _{t \rightarrow T^{-}} \int_{M} \tau h\left(2 \Delta f-|\nabla f|^{2}+\mathcal{R}\right) u d V_{t} \leq \frac{n}{2} h(x, T) .
$$

Proof. Let $\alpha>1, \delta, \epsilon>0$ be constants to be chosen later. By Lemmas 7.3 and 7.4 :

$$
\begin{gathered}
\int_{M} \tau h\left(2 \Delta f-|\nabla f|^{2}+R\right) u d V_{t}=\int_{M} \tau h\left(-2 \Delta u+\frac{|\nabla u|^{2}}{u}+R u\right) d V_{t} \\
\leq \tau(1+4 \delta)\left[\int_{M} \frac{|\nabla u|^{2}}{u} h d V_{\tau}+C_{1}\right]+\tau \int_{M} h u \mathcal{R} d V_{t} \\
\leq \tau \frac{1+4 \delta}{(1-2 \alpha \delta)}\left[C_{2}+\frac{(n+\epsilon) \alpha^{2}}{2 \tau} \int_{M} u h d V_{\tau}\right]+\tau C_{3}
\end{gathered}
$$

where $C_{1}-C_{3}$ are constants independent of $\tau$, provided $0<\tau<\frac{T}{2}$. Here we have used the fact that $h$ is bounded and $\int_{M} u d V_{t}$ is uniformly bounded independent of $\tau$ by Lemma 5.1. Choose $\delta$ small such that $1-2 \alpha \delta>0$. Then

$$
\begin{aligned}
\limsup _{t \rightarrow T^{-}} \int_{M} \tau h\left(2 \Delta f-|\nabla f|^{2}+\mathcal{R}\right) u d V_{\tau} \\
\leq \frac{(n+\epsilon)(1+4 \delta) \alpha^{2}}{(1-2 \alpha \delta)} \limsup _{t \rightarrow T^{-}} \int_{M} u h d V_{t} \\
=\frac{(n+\epsilon)(1+4 \delta) \alpha^{2}}{2(1-2 \alpha \delta)} h(p, T)
\end{aligned}
$$

where we have used the fact that $u \rightarrow \delta_{p}$ as $t \rightarrow T^{-}$and that $h$ is smooth and bounded. Let $\alpha \rightarrow 1, \epsilon, \delta \rightarrow 0$, the result follows.

Lemma 7.6.

$$
\limsup _{t \rightarrow T^{-}} \int_{M} f u h d V_{t} \leq \frac{n}{2} h(x, T)
$$


Proof. Let $\delta>0$ be fixed and choose $C_{1}>0$ such that $C_{1}^{-1} \tau^{\frac{n}{2}} \leq$ $V_{p}^{t}(\sqrt{\tau}) \leq C_{2} \tau^{\frac{n}{2}}$ for any $\tau \in[0, \delta]$ where $\tau=T-t$.

i) We claim that, for any $\epsilon>0$, there is a constant $A>0$, such that

$$
\int_{M \backslash B_{p}^{t}(A \sqrt{\tau})} f h u d V_{t} \leq \epsilon
$$

for any $\tau \in(0, \delta]$.

By Corollary 5.2, we have

$$
u(x, \tau) \leq \frac{C_{1}}{V_{p}^{t}(\sqrt{\tau})} e^{-\frac{r_{t}^{2}(x, p)}{D \tau}}
$$

for some positive constant $C_{2}$ and $D$. Then

$$
\begin{aligned}
& \int_{M \backslash B_{p}^{t}(A \sqrt{\tau})} f h u d V_{t}=\int_{M \backslash B_{p}^{t}(A \sqrt{\tau})} 2 h u \log \frac{\left(\frac{1}{4 \pi \tau}\right)^{\frac{n}{4}}}{\sqrt{u}} d V_{t} \\
& \leq \int_{M \backslash B_{p}^{t}(A \sqrt{\tau})} 2 h u \cdot \frac{\left(\frac{1}{4 \pi \tau}\right)^{\frac{n}{4}}}{\sqrt{u}} d V_{t} \\
& \leq \frac{C_{3}}{\tau^{\frac{n}{4}}} \int_{M \backslash B_{p}^{t}(A \sqrt{\tau})} \sqrt{u} d V \\
& \leq \frac{C_{4}}{\tau^{\frac{n}{2}}} \int_{M \backslash B_{p}^{t}(A \sqrt{\tau})} e^{-\frac{r_{t}^{2}(x, p)}{2 D \tau}} d V_{t}(x) \\
& =\frac{C_{4}}{\tau^{\frac{n}{2}}} \int_{A \sqrt{\tau}}^{\infty} e^{-\frac{r^{2}}{2 D \tau}} A_{p}^{t}(r) d r \\
& =\frac{C_{4}}{\tau^{\frac{n}{2}}}\left(\left[e^{-\frac{r^{2}}{2 D \tau}} V_{p}^{t}(r)\right]_{A \sqrt{\tau}}^{\infty}+\int_{A \sqrt{\tau}}^{\infty} V_{p}^{t}(r) e^{-\frac{r^{2}}{2 D \tau}} d\left(\frac{r^{2}}{2 D \tau}\right)\right) \\
& \leq C_{5} \int_{A \sqrt{\tau}}^{\infty} \tau^{-\frac{n}{2}} \sinh ^{n-1}\left(C_{6} r\right) e^{-\frac{r^{2}}{2 D \tau}} d\left(\frac{r^{2}}{\tau}\right) \\
& \leq C_{7} \int_{A \sqrt{\tau}}^{\infty}\left(\frac{r}{\sqrt{\tau}}\right)^{n} e^{C_{6} r} e^{-\frac{r^{2}}{2 D \tau}} d\left(\frac{r^{2}}{\tau}\right) \\
& =C_{7} \int_{A^{2}}^{\infty} \rho^{\frac{n}{2}} e^{-\frac{\rho}{2 D}} e^{C_{6} \sqrt{\rho \tau}} d \rho \\
& \leq C_{7} \int_{A^{2}}^{\infty} \rho^{\frac{n}{2}} e^{-\frac{\rho}{4 D}} d \rho \quad\left(\text { if } A \geq 4 D C_{6} \sqrt{\delta}\right) \\
& \leq \epsilon \text { (if we choose } A \text { large enough), }
\end{aligned}
$$

where $C_{1}-C_{7}$ are constants independent of $A$ and $\epsilon$. Here we have used the following facts: $\log x \leq x ; h$ and $u$ are positive and $h$ is bounded; 
$V_{p}^{t}(r) \leq C(n) \sinh ^{n}\left(C_{6} r\right)$ by volume comparison; $\sinh \left(C_{6} r\right) / r \leq C e^{C_{6} r}$ for some constant $C$ depending only on $C_{6}$.

ii) By the asymptotic behavior of the heat kernel, see [12 for example, there is an open neighborhood $U$ of $p$, some positive constants $\tau_{0}$ and $C_{6}$ and a positive function $u_{0} \in C^{\infty}\left(U \times\left[0, \tau_{0}\right]\right)$ with $u_{0}(p, 0)=1$, such that

$$
\left|u-\frac{1}{(4 \pi \tau)^{\frac{n}{2}}} e^{-\frac{r_{t}^{2}(x, p)}{4 \tau}} u_{0}(x, \tau)\right| \leq C_{6} \tau^{1-\frac{n}{2}}
$$

for any $x \in U$ and $\tau \in\left(0, \tau_{0}\right]$. Hence, for any $x \in B_{p}^{t}(A \sqrt{\tau})$ when $\tau$ is small,

$$
\begin{aligned}
u & \geq \frac{1}{(4 \pi \tau)^{\frac{n}{2}}} e^{-\frac{r_{t}^{2}(x, p)}{4 \tau}} u_{0}(x, \tau)-C_{6} \tau^{1-\frac{n}{2}} \\
& \geq \frac{1}{(4 \pi \tau)^{\frac{n}{2}}} e^{-\frac{r_{t}^{2}(x, p)}{4 \tau}}\left(1-\frac{C_{7} \tau}{u_{0}(x, \tau)} e^{\frac{r_{t}^{2}(x, p)}{4 \tau}}\right) u_{0}(x, \tau) \\
& \geq \frac{1}{(4 \pi \tau)^{\frac{n}{2}}} e^{-\frac{r_{t}^{2}(x, p)}{4 \tau}}\left(1-C_{8} \tau\right) u_{0}(x, \tau)
\end{aligned}
$$

where all the constants are independent of $\tau$. So

$$
f(x, t) \leq \frac{r_{t}^{2}(x, p)}{4 \tau}-\log \left(1-C_{8} \tau\right)-\log u_{0}(x, \tau)
$$

for any $x \in B_{p}^{t}(A \sqrt{\tau})$ when $\tau$ is small enough.

Hence

$$
\begin{aligned}
& \int_{B_{p}^{t}(A \sqrt{\tau})} f h u d V_{t} \\
\leq & \int_{B_{p}^{t}(A \sqrt{\tau})}\left(\frac{r_{t}^{2}(x, p)}{4 \tau}-\log \left(1-C_{8} \tau\right)-\log u_{0}(x, \tau)\right) h u d V_{t} \\
\leq & \int_{B_{p}^{t}(A \sqrt{\tau})} \frac{r_{t}^{2}(x, p)}{4 \tau} h u d V_{t}+C_{9} \tau \\
= & \int_{B_{p}^{t}(A \sqrt{\tau})} \frac{r_{t}^{2}(x, p)}{4 \tau}\left(\frac{1}{(4 \pi \tau)^{\frac{n}{2}}} e^{-\frac{r_{t}^{2}(x, p)}{4 \tau}} u_{0}(x, \tau)+C_{6} \tau^{1-\frac{n}{2}}\right) h d V_{t}+C_{9} \tau \\
\leq & \int_{B_{p}^{t}(A \sqrt{\tau})} \frac{r_{t}^{2}(x, p)}{4 \tau} \frac{1}{(4 \pi \tau)^{\frac{n}{2}}} e^{-\frac{r_{t}^{2}(x, p)}{4 \tau}} u_{0}(x, \tau) h d V_{t} \\
+ & C_{6} \int_{B_{p}^{t}(A \sqrt{\tau})} \frac{r_{t}^{2}(x, p)}{\tau^{\frac{n}{2}}} h d V_{t}+C_{9} \tau \\
= & \frac{1}{(4 \pi \tau)^{\frac{n}{2}}} \int_{0}^{A \sqrt{\tau}} \frac{r^{2}}{4 \tau} e^{-\frac{r^{2}}{4 \tau}} A_{p}^{t}(r) \tilde{h}(r, t) d r+C_{10} \tau
\end{aligned}
$$


where

$$
\tilde{h}(r, t)=\frac{1}{A_{p}^{t}(r)} \int_{\partial B_{p}^{t}(r)} h u_{0} d S_{t}
$$

Hence

$$
\begin{aligned}
& \int_{B_{p}^{t}(A \sqrt{\tau})} f h u d V_{t} \\
\leq & \frac{1}{2 \pi^{\frac{n}{2}}} \int_{0}^{\frac{A^{2}}{4}} \rho^{\frac{n}{2}} e^{-\rho} \frac{A_{p}^{t}(2 \sqrt{\tau \rho})}{(2 \sqrt{\tau \rho})^{n-1}} \tilde{h}(2 \sqrt{\tau \rho}, t) d \rho+C_{10} \tau
\end{aligned}
$$

where $C_{9}$ and $C_{10}$ are both independent of $\tau$.

Note that

$$
\frac{A_{p}^{t}(2 \sqrt{\tau \rho})}{(2 \sqrt{\tau \rho})^{n-1}} \rightarrow \alpha_{n-1} \quad \text { uniformly for } \rho \in\left[0, \frac{A^{2}}{4}\right] \text { as } \tau \rightarrow 0^{+},
$$

where $\alpha_{n-1}$ means the volume of the the standard sphere of dimension $n-1$. Moreover

$$
\begin{aligned}
& \tilde{h}(2 \sqrt{\tau \rho}, t) \\
= & \frac{1}{A_{p}^{t}(2 \sqrt{\tau \rho})} \int_{\partial B_{p}^{t}(2 \sqrt{\tau \rho})} h u_{0} d S_{t} \rightarrow h(p, T) u_{0}(p, 0) \\
= & h(p, T) \text { uniformly for } \rho \in\left[0, \frac{A^{2}}{4}\right] \text { as } \tau \rightarrow 0^{+} .
\end{aligned}
$$

So,

$$
\begin{aligned}
& \lim _{\sup _{\tau \rightarrow 0^{+}}} \int_{B_{p}^{t}(A \sqrt{\tau})} f h u d V_{t} \\
\leq & \frac{\alpha_{n-1} h(p, T)}{2 \pi^{\frac{n}{2}}} \Gamma\left(\frac{n}{2}+1\right) \\
= & \frac{n \omega_{n}}{2} \cdot \frac{\Gamma\left(\frac{n}{2}+1\right)}{\pi^{\frac{n}{2}}} h(p, T) \\
= & \frac{n}{2} h(p, T)
\end{aligned}
$$

where $\omega_{n}$ means the volume of the unit ball in $\mathbb{R}^{n}$.

The lemma follows from i) and ii).

Proof of Theorem 7.1 (iii). The result follows from Lemmas 7.5 and7.6. the fact that $u \rightarrow \delta_{p}$ as $t \rightarrow T^{-}$and that $h$ is smooth and bounded.

Corollary 7.1. $v(x, t) \leq 0$ on $M \times(0, T]$. 


\section{A pSEudolocality THEOREM}

In this section, we will extend Perelman's pseudolocality theorem to complete noncompact manifolds. We will prove the following:

Theorem 8.1. Let $n$ be fixed. There exist $\delta, \epsilon>0$ with the following property:

Suppose $g(x, t)$ is a smooth complete noncompact solution of the Ricci flow with bounded curvature on $M^{n} \times\left[0, \epsilon^{2}\right]$. Suppose at some point $x_{0} \in M$ the isomperimetric constant in $B_{0}\left(x_{0}, 1\right)$ is larger than $(1-\delta) c_{n}$, where $c_{n}$ is the isoperimetric constant of $\mathbb{R}^{n}$, and $R(x, 0) \geq-1$ for all $x \in B_{0}\left(x_{0}, 1\right)$. Then $|R m(x, t)| \leq t^{-1}+\epsilon^{-2}$ for $0<t \leq \epsilon^{2}$ and $x \in B_{t}\left(x_{0}, \epsilon\right)$.

By the result of [31], we may assume that the covariant derivatives of the curvature are uniformly bounded in spacetime. The proof is similar to the case for compact manifolds using the estimates obtained in previous sections. See [26, 16, 7, 28]. For the sake of completeness, we will sketch the proof.

Suppose this is not true. Then we can find $\left(M_{i}, g_{i}(t)\right), \delta_{i}, \epsilon_{i}>0$ with $\delta_{i}, \epsilon_{i} \rightarrow 0$ and $p_{i} \in M_{i}$ satisfying the following:

(b1) $g_{i}(t)$ is a smooth solution of the Ricci flow on $\left[0, \epsilon_{i}^{2}\right]$ with bounded $\left|\nabla^{k} R m\right|$ on $M_{i} \times\left[0, \epsilon_{i}^{2}\right]$ for all $k \geq 0$.

(b2) The isomperimetric constant in $B_{0}^{(i)}\left(p_{i}, 1\right)$ is larger than (1$\left.\delta_{i}\right) c_{n}$.

(b3) There exist $0<t_{i} \leq \epsilon_{i}^{2}$, and $x_{i} \in B_{t_{i}}^{(i)}\left(p_{i}, \epsilon_{i}\right)$ and $\left|R m\left(x_{i}, t_{i}\right)\right| \geq$ $t_{i}^{-1}+\epsilon_{i}^{2}$.

Let $A_{i}=1 / 1000 n \epsilon_{i}$. By Claims 1 and 2 in [26], see also [16, 7, 28]: We can find $\bar{x}_{i}, \bar{t}_{i}$ with $0<\bar{t}_{i} \leq \epsilon_{i}^{2}$ and $\bar{x}_{i} \in B_{\bar{t}_{i}}^{(i)}\left(p_{i},\left(2 A_{i}+1\right) \epsilon_{i}\right)$ satisfying the following:

(c1) $Q_{i}=\left|R m\left(\bar{x}_{i}, \bar{t}_{i}\right)\right| \geq \frac{1}{\bar{t}_{i}}$, and if

$$
\bar{t}_{i}-\frac{1}{2} Q_{i}^{-1} \leq t \leq \bar{t}_{i}, d_{\bar{t}_{i}}\left(x, \bar{x}_{i}\right) \leq \frac{1}{10} A_{i} Q_{i}^{-\frac{1}{2}}
$$

then

$$
|R m(x, t)| \leq 4\left|R m\left(\bar{x}_{i}, \bar{t}_{i}\right)\right| .
$$

Consider the rescaled flows: $\widehat{g}_{i}(t)=Q_{i} g_{i}\left(\bar{t}_{i}+Q_{i}^{-1} t_{i}\right)$. Then $\widehat{g}_{i}$ satisfies the Ricci flow equation on $M_{i} \times\left[-\frac{1}{2}, 0\right]$ with bounded $\left|\nabla^{k} R m\right|$. Moreover, the following are true:

(d1) $\left|\operatorname{Rm}\left(\bar{x}_{i}, 0\right)\right|=1$.

(d2) If

$$
-\frac{1}{2} \leq t \leq 0, d_{0}\left(x, \bar{x}_{i}\right) \leq \frac{1}{10} A_{i}
$$


then

$$
|R m(x, t)| \leq 4 .
$$

Let $u_{i}$ be the fundamental solution of the conjugate heat equation to the flow $\widehat{g}_{i}$ : $-\left(u_{i}\right)_{t}-\Delta u_{i}+R_{i} u_{i}=0, \lim _{t \rightarrow 0} u=\delta_{\bar{x}_{i}}$. Let $v_{i}$ be the corresponding LYH Harnack expression defined in (7.4) with $\tau=-t$. Then $v_{i} \leq 0$ by Corollary 7.1 .

Case 1: Suppose the injectivity radius at $\bar{x}_{i}$ at $t=0$ are uniformly bounded from below. Because of (d2) and the Ricci flow equation, we know that the injectivity radius of $\bar{x}_{i}$ are uniformly bounded from below at $t=-\frac{1}{2}$. Since $A_{i} \rightarrow \infty$, by the compactness result of Ricci flow [13, 17, for any sequence, we can find a subsequence of $\widehat{g}_{i}$ which converge, still denoted by $\hat{g}_{i}$. Namely, there is $(M, p, g(t))$ with $g(t)$ being a solution of the Ricci flow on $\left[-\frac{1}{2}, 0\right]$, and an exhaustion $U_{i}$ of $M$, diffeomorphisms $\Phi_{i}: U_{i} \rightarrow M_{i}$ with the following properties:

(e1) $\Phi_{i}(p)=\bar{x}_{i}$.

(e2) $\Phi_{i}^{*} \hat{g}_{i}$ converges in $C^{\infty}$ sense to $g$ on $M \times\left(-\frac{1}{2}, 0\right)$.

(e3) The curvature of $g(t)$ is bounded by 4 .

(e4) There exists $0>t_{0}>-\frac{1}{2}$ such that $|R m(p, t)| \geq \frac{1}{2}$ for all $t>t_{0}$.

Note that (e4) is a consequence of $(\mathrm{d} 1),(\mathrm{d} 2),(\mathrm{e} 2)$, local derivatives bound for the curvature tensor and the evolution equation of the curvature tensor.

\section{Lemma 8.1.}

(f1) $\Phi_{i}^{*} u_{i}$ subconverge on $M \times\left(-\frac{1}{2}, 0\right)$ to a solution of

$$
u_{\tau}-\Delta u+R u=0 \text {. }
$$

(f2) $u>0$, and if $v$ is the LYH Harnack expression defined in (7.4) corresponding to $u$, then

$$
v_{\tau}-\Delta v-R v=-2 \tau\left|R_{i j}+\nabla_{i} \nabla_{j} f-\frac{1}{2 \tau} g_{i j}\right|^{2}
$$

where $f$ is given by $u=e^{-f} /(4 \pi \tau)^{\frac{n}{2}}$. Moreover, $v \leq 0$.

Proof. We first prove (f1). By Lemma 5.1, $\int_{M_{i}} u_{i} d V_{t}^{(i)}=1$ for all $i$ and $\tau$. Since $u_{i}>0$ for $\tau>0$, by $(\mathbf{e} 2)$ and the proofs of Corollaries 4.1 and 4.3 , we conclude that $u_{i}$ are locally uniformly bounded. By $(\mathbf{e} 2)$, it is easy to see that (f1) is true. Note that we can construct the function $\rho$ for $(M, g(t))$ as in the proof of Lemma 4.1 and use this to prove a result similar to Corollary 4.1 for $u_{i}$ by $(\mathbf{e} 2)$. See also Remark 4.1,

Next we want to prove (f2). By the proof of Lemma 5.4, for a fixed but small neighborhood $U$ of $p$ there is $c>0$ and $\tau>0$, such that $\int_{U} u_{i} d V_{t}^{(i)} \geq c$ for all $i$. So $u>0$ by the fact that $\phi_{i}^{*} \widehat{g}_{i}$ converges to $g$, 
(f1) and the maximum principle. The rest of the lemma follows from (7.7).

Lemma 8.2. With the same notation as in Lemma 8.1, for any $0<$ $\tau_{0}<\frac{1}{2}$ we have

$$
\int_{B_{\tau_{0}}\left(p, \sqrt{\tau_{0}}\right)} v d V_{-\tau_{0}}<0
$$

Proof. Suppose $\int_{B_{\tau_{0}}\left(p, \sqrt{\tau_{0}}\right)} v d V_{-\tau_{0}}=0$, then $v=0$ in $B_{\tau_{0}}\left(p, \sqrt{\tau_{0}}\right)$. Let $h_{0}$ be a nonnegative smooth function with support in $B_{\tau_{0}}\left(p, \sqrt{\tau_{0}}\right)$ which is positive somewhere. Then for $i$ sufficiently large, we may also consider $h_{0}$ to be a smooth function with compact support in $M_{i}$. Now solve the forward heat equation with initial data $\left.h_{i}\right|_{t=-\tau_{0}}=h_{0}$ on $M_{i} \times\left[-\tau_{0}, 0\right)$. Then since $h_{0}$ is bounded, the $h_{i}$ 's are also uniformly bounded in space time. We may thus assume that $h_{i} \rightarrow h$ which solves the heat equation in $(M, g(t))$ and the convergence is uniform on compact sets of $(x, t) \in$ $M \times\left[-\tau_{0}, 0\right)$. By Theorem 7.1(ii), for $0<\tau<\tau_{0}$

$$
\int_{M_{i}} v_{i}(x,-\tau) h_{i}(x,-\tau) d V_{-\tau}^{(i)} \geq \int_{M_{i}} v_{i}\left(x,-\tau_{0}\right) h_{0}(x) d V_{-\tau_{0}}^{(i)}
$$

where $v_{i}$ is the LYH Harnack expression for $v_{i}$. Since $v_{i} \leq 0$ by Corollary 7.1 and since $h_{0}$ is a fixed function with compact support, let $i \rightarrow \infty$, we can conclude for any compact set $K$ in $M$,

$$
\int_{K} v(x,-\tau) h(x,-\tau) d V_{-\tau} \geq \int_{M} v\left(x,-\tau_{0}\right) h_{0}(x) d V_{-\tau_{0}}=0
$$

Since $v \leq 0$ and $h>0$ for $t>-\tau_{0}$, we have $v=0$ for $0<\tau<\tau_{0}$. By (f2) we have

$$
R_{i j}+f_{i j}-\frac{1}{2 \tau} g_{i j}=0 .
$$

for $\tau<\tau_{0}$. Since the curvature is uniformly bounded, for any $0<\tau<$ $\tau_{0},|\nabla f|$ is at most linear growth. From this one can prove that the vector field $Y_{t}=\left(1-\frac{1}{\tau} t\right)^{-1} \nabla f(-\tau)$ can be integrated from 0 to $t$ as long as $1-\frac{1}{\tau} t>0$ which defines a diffeomorphism $\psi_{t}$.

Then the flow $\widetilde{g}(t)=\left(1-\frac{1}{\tau_{1}} t\right) \psi_{t}^{*}\left(g\left(-\tau_{1}\right)\right)$ is a solution of the Ricci flow on $\left[0, \tau_{1}\right)$ with initial data $g\left(-\tau_{1}\right)$ [6, p. 22-23]. Now $g(t-\tau)$ for $0 \leq t<\tau$ is also such solution. Since the curvatures are bounded for both flows, by the uniqueness result of [5], they are the same. However, by (e4) the curvature of $\left(1-\frac{1}{\tau} t\right) \psi_{t}^{*}\left(g\left(-\tau_{1}\right)\right)$ blows up near $t=\tau$ and the curvature of $g(t-\tau)$ are uniformly bounded, so this is impossible. 
Case 2: Suppose the injectivity radii $a_{i}$ at $\bar{x}_{i}$ at $t=0$ tend to zero. We further rescale the metrics: Let $\hat{\hat{g}}_{i}(t)=a_{i}^{-1} \hat{g}_{i}\left(a_{i} t\right)$. Then it is defined on $\left[-\frac{1}{2} a_{i}^{-1}, 0\right]$ with the following properties:

(g1) $\left|\operatorname{Rm}\left(\bar{x}_{i}, 0\right)\right|=a_{i}$.

(g2) If

$$
-\frac{1}{2} a_{i}^{-1} \leq t \leq 0, d_{0}\left(x, \bar{x}_{i}\right) \leq \frac{1}{10} A_{i} \cdot a_{i}^{-\frac{1}{2}}
$$

then

$$
|R m(x, t)| \leq 4 a_{i} .
$$

(g3) There exists $t_{0}<0$ such that the injectivity radius of $\bar{x}_{i}$ at time $t_{0}$ is less than 2 .

(g3) can be proved by the fact that $a_{i} \rightarrow 0$ and the injectivity radius bound in 3 .

As before, we can find a limit metric and flow $(M, g(t), p)$ on $(-\infty, 0]$ with the following properties:

(h1) $g(t)$ is flat for all $t$.

(h2) The injectivity radius of $p$ at time $t_{0}$ is less than or equal to 2 .

Let $v$ and $f$ as before.

Lemma 8.3. For any $0<\tau_{0}<\infty$ we have

$$
\int_{B_{\tau_{0}}\left(p, \sqrt{\tau_{0}}\right)} v d V_{-\tau_{0}}<0 .
$$

Proof. As before, if this is not true, then one can prove that

$$
R_{i j}+f_{i j}-\frac{1}{2 \tau} g_{i j}=0 .
$$

for $0<\tau<\tau_{0}$. Since curvature is bounded, there is $0<\tau_{1}<\tau_{0}$ such that $f_{i j} \geq g_{i j}$. One can prove that

$$
g\left(t-\tau_{1}\right)=\left(1-\frac{1}{\tau_{1}} t\right) \psi_{t}^{*}\left(g\left(-\tau_{1}\right)\right)
$$

The function $f$ is an exhaustion function, see [1] for example. So there is a point such that $\nabla f=0$. Hence we can find a point $q$ which is a fixed point of $\psi_{t}$. Hence the injectivity radius of $q$ with respect to $\psi_{t}^{*}\left(g\left(-\tau_{1}\right)\right)$ is independent of $t$. Note that $M$ is flat but is not $\mathbb{R}^{n}$ by (h2), and so the injectivity radius of $q$ is finite. On the other hand, $g(t)=g\left(-\tau_{1}\right)$ because of (h1) and the fact that $g$ satisfies the Ricci flow equation. Hence the injectivity radius of $q$ of $g(t)$ is independent of $t$. This is impossible as $t \rightarrow \tau_{1}$ by (8.1). 
From these, it is easy to see that Claim 3 in [26, §10] is also true. One can conclude that Theorem 8.1 is true by the argument of [26], see also [16, 7, 20, 28].

\section{SINGULARITY FORMATION AND LONGTIME EXISTENCE}

We now apply the pseudolocality result to describe where singularities to the Ricci flow can form under certain assumptions. More precisely, in Theorem 9.1 we prove that any finite time singularities of the Ricci flow (9.1) under Assumption 1 below must form within a compact set. In Theorem 9.2 we apply this result to complete non-negatively curved Kählermanifolds and prove a long time existence result for the Kähler-Ricci flow.

Consider the Ricci flow

$$
\begin{cases}\frac{d}{d t} g & =-2 R c \\ g(t) & =g .\end{cases}
$$

on a complete non-compact Riemannian manifold. Let us make the following assumption

Assumption 1. Let $(M, g)$ be a complete non-compact Riemannian manifold of dimension $n$ such that

(1) $|R m(x)| \rightarrow 0$ as $d(x, p) \rightarrow \infty$ for some fixed point $p$

(2) the injectivity radius inj $(M, g)$ of $(M, g)$ is bounded from below.

Lemma 9.1. Let $(M, g)$ be as in Assumption 1 and let $(M, g(t))$ be the corresponding maximal solution to the Ricci flow (9.1) on $M \times[0, T$ ). For any $0<\delta<1$, there exists $r>0$ with the following property: Given any $t^{\prime}<T$ there exists $0<d^{\prime}<\infty$ such that for any $x \in M$ and $0<t \leq t^{\prime}$ with $d_{t}(x, p) \geq d^{\prime}$, we have

$$
\operatorname{Vol}_{t}(\partial \Omega)^{n} \geq(1-\delta) c_{n} \operatorname{Vol}_{t}(\Omega)^{n-1}
$$

for any $\Omega \subset B_{t}(x, r)$.

Proof. Let $0<\delta<1$ be given and let $r_{0}=\operatorname{inj}(M, g(0)) / 2$. By conditions (1) and (2) in Assumption 1, we can find some $0<d_{0}<\infty$ such that for any $x$ where $d_{0}(x, p) \geq d_{0}$, we have

$$
\operatorname{Vol}_{0}(\partial \Omega)^{n} \geq\left(1-\frac{\delta}{2}\right) c_{n} \operatorname{Vol}_{0}(\Omega)^{n-1}
$$

for any $\Omega \subset B_{0}\left(x, r_{0}\right)$.

By Theorem 18.2 in [14, given $t^{\prime}<T$ and any $\eta>0$ there exists some compact $S \subset M$ such that

$$
|R m(x, t)| \leq \eta
$$


for all $(x, t) \in(M \backslash S) \times\left[0, t^{\prime}\right]$.

By choosing $\eta$ sufficiently small we see from (9.1), (9.2) and (9.3) that we may choose some $d^{\prime}>d_{0}$ such that for any $x$ where $d_{t}(x, p) \geq d^{\prime}$, we have

$$
\operatorname{Vol}_{t}(\partial \Omega)^{n} \geq(1-\delta) c_{n} \operatorname{Vol}_{t}(\Omega)^{n-1}
$$

for any $\Omega \subset B_{t}\left(x, \frac{r_{0}}{2}\right)$. Thus $\frac{r_{0}}{2}$ satisfies the conclusion of the Lemma.

Theorem 9.1. Let $(M, g)$ satisfy Assumption 1 and let $(M, g(t))$ be the corresponding maximal solution to the Ricci flow (9.1) on $M \times[0, T)$. Then either $T=\infty$ or there exists some compact set $S \subset M$ with the property that $|R m(x, t)|$ is uniformly bounded on $(M \backslash S) \times[0, T)$.

Proof. Assume that $T<\infty$ and let $g(t)$ be a maximal solution to (9.1) on $M \times[0, T)$. Thus there exists sequences $x_{i} \in M$ and $t_{i} \rightarrow T$ such that $\left|R m\left(x_{i}, t_{i}\right)\right| \rightarrow \infty$ as $i \rightarrow \infty$. We will show that there exists some compact $S \subset M$ such that every such sequence $x_{i}$ must be contained inside $S$. $S$ will then clearly satisfy the conclusion of the Theorem.

Suppose there is a sequence $\left(x_{i}, t_{i}\right)$ satisfying the above condition and $d_{0}\left(p, x_{i}\right) \rightarrow \infty$. Let $\delta, \epsilon$ be as in Theorem 8.1. For such $\delta$, let $r$ be as in Lemma 9.1. By rescaling our solution $g(t)$ in both time and space, we may assume that $r=1$ (without affecting $\delta$ ). Now let $t^{\prime}=T-\epsilon^{2}$, and choose $d^{\prime}$ as in Lemma 9.1. Then by Theorem 18.2 in [14, we may assume $d^{\prime}$ sufficiently large so that $R\left(y, t^{\prime}\right) \geq=-1$ for all $y \in B_{t^{\prime}}(y, x)$ where $d_{t^{\prime}}(x, p) \geq 0$. We may assume that $\epsilon>0$ is small enough such that $t^{\prime}>0$.

Let $\eta_{k} \rightarrow 0$ and let $\tau_{k}=t^{\prime}-\eta_{k}>0$ and $g_{k}(t)=g\left(\tau_{k}+t\right)$. Then $g_{k}(t)$ is well defined on $\left[0, \epsilon^{2}\right]$. By [31, 14], we know that for each $k$ the curvature tensor of $g_{k}(t)$ together with its derivatives are uniformly bounded in $\left[0, \epsilon^{2}\right]$. Let $i_{0}$ be large enough such that if $i \geq i_{0}$, then $d_{t}\left(x_{i}, p\right) \geq d^{\prime}$ for all $0<t \leq t^{\prime}$.

By Theorem 8.1, we have

$$
\left|R m_{k}\left(x_{i}, t\right)\right| \leq t^{-1}+\epsilon^{-2}
$$

for all $i \geq i_{0}$ and $0 \leq t \leq \epsilon^{2}$. Here $R m_{k}$ is the curvature tensor for $g_{k}$. Now $R m\left(x_{i}, t_{i}\right)=R m_{k}\left(x_{i}, t_{i}-\tau_{k}\right)$. We have

$$
t_{i}-\tau_{k}=t_{i}-t^{\prime}+\eta_{k}=t_{i}-T+\epsilon^{2}+\eta_{k} .
$$

Hence for fixed $i \geq i_{0}$ such that $T-t_{i} \leq \epsilon^{2}$, we have $0 \leq t_{i}-\tau_{k} \leq \epsilon^{2}$ for $k$ sufficiently large.

So

$$
\left|R m\left(x_{i}, t_{i}\right)\right|=\left|R m_{k}\left(x_{i}, t_{i}-\tau_{k}\right)\right| \leq\left(t_{i}-\tau_{k}\right)^{-1}+\epsilon^{-2} .
$$


Now letting $k \rightarrow \infty$ and then letting $i \rightarrow \infty$, we have

$$
\limsup _{i \rightarrow \infty}\left|R m\left(x_{i}, t_{i}\right)\right| \leq 2 \epsilon^{-2} .
$$

This contradicts our initial assumption, and thus completes the proof of the Theorem.

Corollary 9.1. Suppose $T<\infty$ in Theorem 9.1. Then $\operatorname{Rm}(x, T) \rightarrow 0$ as $x \rightarrow \infty$ in the sense that: given any $\epsilon>0$, we may choose $S$ such that $|\operatorname{Rm}(x, t)| \leq \epsilon$ for all $(x, t) \in S^{c} \times[0, T)$.

Proof. Assume the Corollary is false. Thus there exits a space time sequence $\left(x_{k}, t_{k}\right)$ such that $d_{0}\left(p, x_{k}\right) \rightarrow \infty, t_{k} \rightarrow T$ and $\left|R m\left(x_{k}, t_{k}\right)\right| \geq$ $C_{1}$ for some $C_{1}>0$.

1. Fix some small $\epsilon_{1}>0$ to be chosen later and let $s_{k}=t_{k}-\epsilon_{1}$. Then by Theorem 9.1 and Theorem 18.2 and 13.1 in [14], we may assume the compact set $S \subset M$ from Theorem 9.1 was chosen sufficiently large so that for some $C_{2}>0$ we have

$$
\left|\frac{d}{d t} R m\left(x_{k}, t\right)\right| \leq C_{2}
$$

for all $k$ sufficiently large and $t \in\left[0, t_{k}\right]$.

2. For $k$ suffuciently large, $s_{k} \in\left[0, T-\epsilon_{1}\right]$. Thus by Theorem 18.2 in [14], given any $\epsilon_{2}>0$ we may assume $S \subset M$ was chosen sufficiently larger still so that

$$
\left|R m\left(x_{k}, s_{k}\right)\right| \leq \epsilon_{2}
$$

for $k$ sufficiently large.

Thus by 1 and 2 , for $k$ sufficiently large we have that

$$
\left|R m\left(x_{k}, t_{k}\right)\right| \leq\left|R m\left(x_{k}, s_{k}\right)\right|+C_{2} \epsilon_{2} \leq \epsilon_{2}+C_{2} \epsilon_{1} .
$$

Note that $\epsilon_{1}, \epsilon_{2}$ were chosen independently of each other, and that $C_{2}$ can be chosen independent of these. Thus for sufficiently small choices of $\epsilon_{1}$ and $\epsilon_{2}$, we arrive at a contradiction. This compeletes the proof by contradiction.

We now apply Theorem 9.1 to the case non-negatively curved Kähler manifolds.

Theorem 9.2. Let $(M, g)$ be a complete non-compact Kähler manifold with non-negative holomorphic bisectional curvature, strictly positive at some point. Then if $(M, g)$ satisfies Assumption 1, the Kähler-Ricci flow has a long time solution $g(t)$ on $M \times[0, \infty)$. 
Proof. Assume the Theorem is false and that $g(t)$ is a maximal solution on $M \times[0, T)$ for $T<\infty$. Let the set $S$ be as in Theorem 9.1. Now define

$$
F(x, t)=\log \frac{\operatorname{det} g(x, t)}{\operatorname{det} g(x, 0)} .
$$

By [34, we know that $g(t)$ also has non-negative holomorphic bisectional curvature and hence $F(x, t) \leq 0$. We claim that $-F(x, t)$ is uniformly bounded on $M \times[0, T)$. If this is true, then the curvature is also uniformly bounded on $M \times[0, T)$ by the argument in [34, $\S 7]$. From this it is easy to see that the solution $g(t)$ can be extended beyond $T$ by [31, 5]. This is a contradiction.

Since the curvature $|R m(x, t)|$ is uniformly bounded in $(M \backslash S) \times[0, T)$ and since

$$
-F(x, t)=\int_{0}^{t} R(x, \tau) d \tau
$$

where $R$ is the scalar curvature, there exists $C_{1}$ such that

$$
0 \leq-F(x, t) \leq C_{1}
$$

for all $(x, t) \in(M \backslash S) \times[0, T)$.

Next we want to prove that there exists $C_{2}$ such that

$$
-F(x, t) \leq C_{2}
$$

for all $(x, t) \in S \times[0, T)$.

Let $\tilde{M}$ be the universal cover of $M$. Then by [24, $\tilde{M}=\tilde{N} \times \tilde{L}$ holomorphically and isometrically where $\tilde{N}$ is compact and $\tilde{L}$ satisfies

$$
\frac{1}{\tilde{V}_{\tilde{o}}(r)} \int_{\tilde{B}_{\tilde{o}}(r)} \tilde{R} \leq \frac{C}{1+\tilde{r}}
$$

where $\tilde{B}_{\tilde{o}}(r)$ is the geodesic ball in $\tilde{L}$ and $\tilde{V}_{\tilde{o}}(r)$ is its volume. Also $\tilde{R}$ is the scalar curvature of $\tilde{L}$. Since $|R m(x)| \rightarrow 0$ as $x \rightarrow \infty$ in $M$, we must have $\tilde{M}=\tilde{L}$.

Suppose there exist sequences $x_{i} \in S$ and $t_{i} \rightarrow T$ such that $F\left(x_{i}, t_{i}\right) \rightarrow$ $-\infty$. By (9.5), we may assume that

$$
m\left(t_{i}\right):=\min _{M} F\left(\cdot, t_{i}\right)=F\left(x_{i}, t_{i}\right)
$$

provided $i$ is sufficiently large. After we lift the Kähler-Ricci flwo to $\tilde{M}$, there is a compact set $\tilde{S}$ in $\tilde{M}$ and $\tilde{x}_{i} \in \tilde{S}$ such that $F\left(\tilde{x}_{i}, t_{i}\right) \rightarrow-\infty$ and $\min _{\tilde{M}} F\left(\cdot, t_{i}\right)=F\left(\tilde{x}_{i}, t_{i}\right)=m\left(t_{i}\right)$. Here $F(\tilde{x}, t)$ is the logarithm of the ratio of volume elements for the flow in $\tilde{M}$. 
Then by the proof of Corollary 2.1 of [25] we have

$$
\begin{aligned}
-m\left(t_{i}\right) & \leq C_{3} \int_{0}^{\sqrt{a t_{i}\left(1-m\left(t_{i}\right)\right)}} \frac{s}{1+s} d s \\
& \leq C_{3} \sqrt{a t_{i}\left(1-m\left(t_{i}\right)\right)}
\end{aligned}
$$

for some positive constants $a, C_{3}$, where we have used the fact that $\tilde{S}$ is compact and (9.7). From this we can see that (9.6) is true. This completes the proof of the claim and the theorem.

\section{REFERENCES}

[1] Chau, A. and Tam, L.-F., On the complex structure of Kähler manifolds with non-negative curvature, J. Differential Geom. 73 (2006), 491-530.

[2] Chau, A. and Tam, L.-F., Non-negatively curved Kähler manifolds with average quadratic curvature decay, to appear in Comm. Anal. Geom.

[3] Cheeger, J., Gromov, M. and Taylor, M., Finite propagation speed, kernel estimate for functions of the Laplace operator and the geometry of complete Riemannian manifolds, J. Differential Geom. 17 (1982), no. 1, 15-53

[4] Chen, B.L. and Zhu, X.P., Volume Growth and Curvature Decay of Positively Curved Kähler manifolds, Q. J. Pure Appl. Math. 1 (2005), no. 1, 68-108. MR2154333.

[5] Chen and Zhu, Uniqueness of the Ricci Flow on Complete Noncompact Manifolds, arXiv: math.DG/0505447

[6] Chow, B. and Knopf, D., The Ricci flow: an introduction Mathematical Surveys and Monographs, 110, American Mathematical Society, Providence, RI, 2004.

[7] Chow, B., Lu, P., and Ni, L., Hamilton's Ricci flow, v. 1 and 2, in preparation.

[8] Davies, E.B., Heat kernels and spectral theory, Cambridge University Press, 1989.

[9] Ecker, K. and Huisken, G., Interior estimates for hypersurfaces moving by mean curvature, Invent.math. 105 (1991), 547-569.

[10] Garofalo, N. and Lanconelli, E., Asymptotic behavior of fundamental solutions and potential theory of parabolic operators with variable coefficients, Math.Ann. 283 (1989),211-239.

[11] Grigor'yan, A., Gaussian upper bounds for the heat kernel on arbitrary manifolds, J. Differential Geometry, 45 (1997), 33-52.

[12] Guenther, C.M., The fundamental solution on Manifolds with time-dependent metrics. J.Geometric Analysis, 12(3) (2002), 425-436.

[13] Hamilton, R., A compactness property for solutions of the Ricci flow. Amer.J.Math. 117 (1995), no.3, 545-572.

[14] Hamilton, R. S., Formation of Singularities in the Ricci Flow, Surveys in differential geometry, Vol. II (1995), 7-136, MR1375255, Zbl 0867.53030. Record 2 of 3

[15] Ito, S., Diffusion equations, Translations of mathematical monographs v. 114, American Mathematical Society, 1992. .

[16] Kleiner, B. and Lott, J., Notes on Perelman's papers, arXiv:math.DG/0605667. 
[17] Kuang, S. and Zhang, Q. S., A gradient estimate for all positive solutions of the conjugate heat equation under Ricci flow, arXiv:math.DG/0611298.

[18] Li, P.; Schoen, R., $L^{p}$ and mean value properties of subharmonic functions on Riemannian manifolds, Acta Math. 153 (1984), no. 3-4, 279-301.

[19] Li, P. and Yau, S.-T., On the parabolic kernel of the Schrödinger operator, Acta Math. 156 (1986) 139-168.

[20] Ni, L., The entropy formula for linear heat equation, J. Geom. Anal. 14 (2004), no. $1,87-100$

[21] Ni, L., Ricci flow and nonnegativity of sectional curvature, Math. Res. Lett. 11 (2004), 883-904.

[22] Ni, L., Ancient solutions to Kähler-Ricci flow, Math. Res. Lett. 12 (2005), 633-653.

[23] Ni, L., A note on Perelman's LYH inequality, arXiv:math.DG/0602337.

[24] Ni, L., and Tam, L.-F., Plurisubharmonic functions and the structure of complete Kähler manifolds with nonnegative curvature, J. Differential Geom. 64 (2003), 457-524.

[25] Ni, L. and Tam, L.-F., Kähler-Ricci flow and the Poincaré-Lelong equation, Comm. Anal. Geom. 12 (2004), 111-141, MR2074873, Zbl 1067.53054.

[26] Perelman, G., The entropy formula for the Ricci flow and its geometric applications, arXiv:math.DG/0211159.

[27] Saloff-Coste, L., Uniformly elliptic operators on Riemannian manifolds, J.Differential Geom. 36 (1992), 417-450.

[28] Sesum, N., Tian, G. and Wong, X., Notes on Perelmans paper on the entropy formula for the Ricci flow and its geometric applications, in preparation.

[29] Shi, W.-X., Ricci deformation of the metric on complete noncompact Riemannian manifolds, J.Differential Geom. 30 (1989), 223-301.

[30] Shi, W.-X., Ricci Flow and the uniformization on complete non compact Kähler manifolds, J.Differential Geom. 45 (1997), 94-220.

[31] Shi, W.-X., Ricci deformation of the metric on complete noncompact Riemannian manifolds, J.Differential Geom. 30 (1989), 223-301.

[32] Shi, W.-X., Ricci deformation of the metric on complete noncompact Kähler manifolds, PhD thesis, Harvard University, 1990.

[33] Shi, W.-X.,Complete noncompact Kähler manifolds with positive holomorphic bisectional curvature, Bull.Amer.Math.Soc. (N.S.) 23 (1990), 437-400.

[34] Shi, W.-X., Ricci Flow and the uniformization on complete non compact Kähler manifolds, J.Differential Geom. 45 (1997), 94-220.

[35] Zhang, Q., Some gradient estimates for the heat equation on domains and for an equation by Perelman, arXiv:math.DG/0605551s. 
Waterloo University, Department of Pure Mathematics, 200 UniVERsity aVenue, Waterloo, ON N2L 3G1, CANADA

E-mail address: a3chau@math.uwaterloo.ca

Department of Mathematics, The Chinese University of Hong Kong, Shatin, Hong Kong, China.

E-mail address: Iftam@math.cuhk.edu.hk

Department of Mathematics, The Chinese University of Hong Kong, Shatin, Hong Kong, China.

E-mail address: cjyumath.cuhk.edu.hk 